\title{
Fonología segmental del chiquitano migueleño Segmental phonology of Migueleño Chiquitano
}

\author{
Andrey Nikulin (10 \\ Universidade de Brasília. Brasília, Distrito Federal, Brasil
}

\begin{abstract}
Resumen: Este artículo describe la fonología segmental del chiquitano migueleño (familia macro-ye), una variedad severamente amenazada hablada en el municipio de San Miguel de Velasco (Santa Cruz, Bolivia). La fonología de esta variedad presenta una serie de procesos tanto léxicos como posléxicos, algunos de los cuales no han sido atestiguados en ninguna otra variedad del chiquitano. Se proponen 21 fonemas consonánticos, de los cuales 7 son marginales: / $\underline{\underline{j}} \subseteq 6$ S/ son demostrablemente derivadas de $|\mathrm{t} \mathrm{p} / \mathrm{k} \times \mathrm{f}|$ subyacentes mediante los procesos morfofonológicos de palatalización y ensordecimiento, / $\mathrm{u} \mathrm{g} /$ resultan de un proceso morfofonológico de epéntesis, mientras que /h/ es de baja frecuencia y alterna con cero. En cuanto al sistema vocálico, se proponen doce fonemas /a + $\varepsilon$ i o u ã ̃ ̃ ̃ i õ ũ/, que se ensordecen en algunos ambientes. En la superficie, las nasales adquieren una fase consonántica si no están adyacentes a / $/$ / o seguidas de /x/, excepto las secuencias tautosilábicas /a ̃̃ oz̃/, que jamás adquieren la fase consonántica. Además, se documenta un proceso que inserta la secuencia [jo] tras una /i/ final.
\end{abstract}

Palabras claves: Chiquitano. Fonología. Palatalización. Ensordecimiento vocálico. Macro-ye.

Abstract: The article contains a description of the segmental phonology of the Migueleño dialect of Chiquitano (Macro-jê), a critically endangered variety spoken in San Miguel de Velasco (Santa Cruz, Bolivia). The phonology of this variety presents a number of processes, both lexical and postlexical, some of which have not been attested in other varieties of Chiquitano. I propose an inventory of 21 consonantal phonemes, including 7 segments whose phonological status is marginal: / $/ \underline{\mathrm{t}} \subseteq \mathbf{c}$ s/ are demonstrably derived from underlying $|\mathrm{t} p / \mathrm{k} \times \mathrm{r}|$ by means of the morphophonological processes of palatalization and devoicing, / $/ \mathrm{u} \mathrm{g} /$ result from a morphophonological process of epenthesis, whereas $/ \mathrm{h} / \mathrm{is}$ of low frequency and alternates with zero. As for the vowels, I propose twelve phonemes /a † $\varepsilon$ i o u ã $\tilde{\ddagger} \tilde{\varepsilon}$ ĩ õ ũ/, which may undergo devoicing in certain environments. Nasal vowels acquire a consonantal phase at surface unless they are adjacent to / $\mathrm{R} /$ or followed by

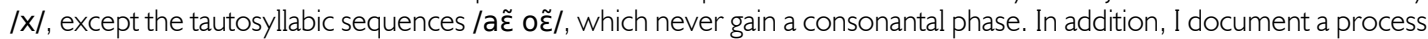
that inserts the sequence [jo] after a word-final /i/.

Keywords: Chiquitano. Phonology. Palatalization. Vowel devoicing. Macro-Jê.

Nikulin, A. (2021). Fonología segmental del chiquitano migueleño. Boletim do Museu Paraense Emílio Goeldi. Ciências Humanas, 16(2), e20200054. doi: 10.1590/2178-2547-BGOELDI-2020-0054

Autor para correspondência: Andrey Nikulin. Universidade de Brasília. Campus Universatário Darcy Ribeiro Asa Norte. Brasília, DF, Brasil. CEP 70910-900 (andre.n.guzman@gmail.com).

Recebido em 27/04/2020

Aprovado em 10/09/2020

Responsabilidade editorial: Hein van der Voort

(c) (i)

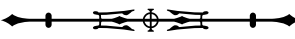




\section{INTRODUCCIÓN}

El chiquitano migueleño (localmente conocido simplemente como 'el dialecto' y autodenominado ózura ['ọtsurąa] 'nuestra habla') es una variedad lingüística severamente amenazada recordada por algunos individuos, en su mayoría ancianos, en el municipio de San Miguel de Velasco, ubicado en la provincia de José Miguel de Velasco del departamento de Santa Cruz, Bolivia. A pesar de que dicha variedad esté estrechamente relacionada a las demás variedades del chiquitano, como el bésiro (nombre dado a la variedad utilizada en la región de Lomerío y en San Javier, la única que posee estatus oficial en Bolivia) y el chiquitano oriental (hablado en las cercanías de San Ignacio de Velasco, San Rafael de Velasco y Santiago de Chiquitos), las diferencias fonológicas, morfológicas, morfosintácticas y léxicas entre ellas son extremadamente numerosas, al punto de que según el criterio de la inteligibilidad mutua el chiquitano migueleño podría considerarse una lengua aparte. En cuanto a la filiación lingüística, el conjunto de las variedades del chiquitano ha sido clasificado como perteneciente a la familia lingüística macro-ye (Adelaar, 2008) o al menos remotamente relacionado a esa familia (Nikulin, 2020a, pp. 179-181).

La documentación del chiquitano migueleño se encuentra en una etapa inicial. La primera obra publicada en o sobre esa variedad es el volumen producido por Parzinger \& Cabildo Indígena de San Miguel de Velasco (2016), en el que se encuentran reunidos los sermones cristianos de la región que vienen siendo transmitidos oralmente y vía cuadernos manuscritos desde la época jesuítica (ver sección 'Perfil sociolingüístico'). Recientemente han salido artículos cortos acerca de algunas particularidades morfológicas y morfofonológicas del dialecto (Nikulin, 2019b, 2019c, 2020b). Además, existen dos manuscritos no publicados, uno de Bas Aguilera (sin fecha) con una colección de frases y vocablos, y otro del presente autor (Nikulin, 2019a), que consiste en un diccionario y un esbozo gramatical del chiquitano migueleño.

Este estudio, cuyo objetivo es proporcionar una descripción fonética y un análisis fonológico del chiquitano migueleño, se basa en los materiales que provienen del trabajo de campo realizado por el autor en cinco visitas (julio de 2017, enero de 2018, junio de 2018, febrero de 2019, julio de 2019) a las comunidades de San Juan de Lomerío y San Miguel de Velasco. Los datos analizados aquí son de dos hablantes (q) de San Juan y de un hablante (đ̋) de San Miguel’

En la sección 'Perfil sociolingüístico', se describe la situación sociolingüística del chiquitano migueleño. La fonología segmental se abordará en las secciones tituladas 'Consonantes' y 'Vocales'. La discusión en las secciones anteriores se resumirá en las 'Conclusiones'. Finalmente, el 'Glosario' contiene una lista de términos del castellano regional con sus definiciones y/o equivalentes en el castellano venezolano y en el portugués.

Se optó por no incluir una discusión detallada de las semejanzas y de las diferencias entre los sistemas fonológicos del chiquitano migueleño y de las demás variedades del chiquitano, como el bésiro (Krüsi \& Krüsi, 1978; Parapaino Castro, 2008; Sans, 2010, 2011, 2013), la variedad de San Rafael de Velasco (Girard, 2012, 2014), la de San Javierito (Ciucci \& Macoñó Tomichá, 2018) y la de Brasil (Santana, 2005, 2012); a ese tema se le dedicará una publicación futura. Tampoco se discutirán los procesos morfofonológicos, que se examinan en una otra publicación (Nikulin, 2020b), ni el acento, que se analizará en una publicación futura.

\footnotetext{
Aunque el chiquitano migueleño posee un sistema de diferencias generolectales, éstas no interactúan con el nivel fonológico, excepto

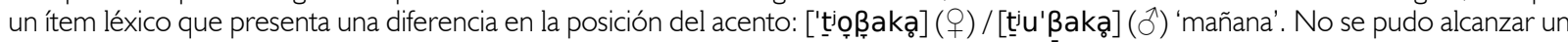
balance de las variables 'género' y 'comunidad de origen', ya que no hay hablantes del género masculino en San Juan. En San Miguel, se han realizado grabaciones con hablantes de ambos géneros, pero apenas uno de los consultantes de San Miguel poseía dicción suficientemente clara para un análisis fonético. Ambos hablantes del género masculino con los que se trabajó fallecieron en 2019.
} 


\section{PERFIL SOCIOLINGÜÍSTICO}

La situación sociolingüística del chiquitano migueleño es crítica, resultado de un cese abrupto de transmisión lingüística de esa variedad ocurrido a mediados del siglo XX. Actualmente la variedad lingüística dominante en San Miguel es el dialecto camba del castellano. En el escenario más optimista, el chiquitano migueleño contaría hoy con menos de 30 hablantes, dispersos por la zona rural del municipio de San Miguel de Velasco. A pesar de que hasta la presente fecha no se haya conducido una investigación sociolingüística detallada, se ha podido obtener la siguiente estimación del número de hablantes en cada comunidad: 4 en Altamira, 2 en San Juan de Lomerío, 2 en San Francisco de Lima, 1 en Santa Rosita de Lomerío, 1 en San José de Corralito y un número desconocido (por lo menos 1) en San Antonio Tacoó. Además de estos, en la zona urbana de San Miguel residen aproximadamente 10 hablantes del chiquitano (incluyendo a la única hablante monolingüe viva de la que se ha tenido noticia). Sin embargo, no se sabe de ningún núcleo familiar o vecinal en el que la lengua originaria siga siendo utilizada regularmente (con la posible excepción de la hablante monolingüe mencionada, que se comunica con su hija semihablante). Como consecuencia de la situación de desuso del chiquitano migueleño, la mayoría (si no la totalidad) de los hablantes admite tener dificultades en cuanto a la producción oral en su lengua materna.

Además de los hablantes plenos, algunas personas retienen un dominio pasivo del chiquitano migueleño. Se trata sobre todo de hijos biológicos o adoptivos de hablantes monolingües ya fallecidos. En su mayoría, estos semihablantes afirman no hablar la lengua, pero por lo menos algunos de ellos pueden comprender fácilmente el chiquitano hablado². Las instancias de producción lingüística de los semihablantes que se han registrado se limitan a palabras aisladas, generalmente adaptadas a la fonética del castellano camba (las sustituciones más comunes son: [i] $\rightarrow[\mathrm{u}]$; [c] $\rightarrow$ [ki]; [ts] $\rightarrow$ [s], [?] $\rightarrow$ cero).

Es necesario subrayar que existe una situación extraordinaria en la que el chiquitano migueleño todavía se emplea con cierta frecuencia. Se trata de la recitación de los sermones cristianos (la población de San Miguel es, en su casi totalidad, católica romana). Actualmente los sermones, cuyos textos vienen siendo transmitidos oralmente y en cuadernos manuscritos, son recitados por los caciques y los maestros de capilla (semihablantes o no hablantes del chiquitano) en determinadas ocasiones, tales como las fiestas religiosas (Parzinger, 2017). Recientemente se publicó una importante recopilación de los sermones de San Miguel (Parzinger \& Cabildo Indígena de San Miguel de Velasco, 2016). Sin embargo, el lenguaje de los sermones no es completamente idéntico al lenguaje utilizado en el habla normal: por ejemplo, la composición morfológica del ítem [tsa'ika] 'mis hijos', omnipresente en los sermones, difiere de aquella de [ $\varepsilon$,tsai'tainkị] 'id.', que ocurre en los datos del autor (la forma [tsa'ika] es fácilmente asociada por los hablantes al lenguaje de los sermones). Es posible que el lenguaje utilizado en los sermones represente una etapa anterior del chiquitano migueleño, hablada todavía en la época jesuítica.

Los no hablantes del chiquitano residentes en el municipio de San Miguel demuestran un interés, aunque moderado, por la revitalización de esa variedad lingüística. Algunos de ellos tienen conocimiento de las diferencias

\footnotetext{
2 Ejemplifico esta afirmación con evidencia anecdótica. El presente autor tuvo la ocasión de trabajar con una hablante anciana del chiquitano migueleño (su consultante principal) en la presencia de su sobrina, huérfana desde muy corta edad y criada por su abuela (que era una hablante competente del chiquitano). En un determinado momento, la consultante, que por su avanzada edad posee una capacidad auditiva reducida, pidió al autor que repitiera la frase que él había acabado de decir (“/tsoĩtsimóka kinána núta?a soźsi | aß̧é?e taríçaa/"). No obstante, antes de que el autor haya podido hacerlo, la sobrina de la consultante repitió, en castellano: "nos sentámos debajo del árbol y había petos, dice", demostrando haber comprendido la frase pronunciada por el autor en chiquitano. Sin embargo, esa sobrina de la consultante afirma que no habla la lengua originaria.
}

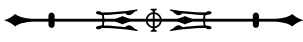


más notables entre las variedades migueleña y lomeriana del chiquitano, como la correspondencia entre [x] en San

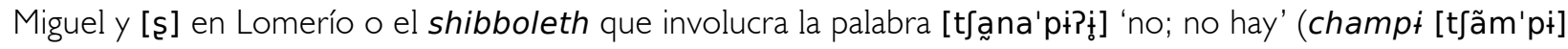
en Lomerío). Actualmente el currículo escolar en la región incluye la enseñanza del bésiro (la variedad lomeriana del chiquitano), aunque los profesores que imparten las clases no son hablantes de esa variedad y poseen una competencia apenas inicial en bésiro ${ }^{3}$. Según las observaciones del autor, los profesores tienen conciencia de que la variedad enseñada no es la originaria de San Miguel, y ha habido algunos intentos de introducir material migueleño en las clases del chiquitano (en un caso, la maestra cuenta con la ayuda del único hablante de la comunidad), que culminaron en la publicación de una edición de un libro didáctico del bésiro (de distribución extremadamente limitada) en la que algunas palabras lomerianas vienen acompañadas de su equivalente migueleño (en una ortografía no estandarizada).

\section{CONSONANTES}

Se postula aquí para el chiquitano migueleño un inventario máximo de 21 fonemas consonánticos, que se encuentran expuestos en la Tabla $1^{4}$.

Tabla 1. Las consonantes del chiquitano migueleño.

\begin{tabular}{c|c|c|c|c|c|c|c}
\hline & Labiales & Dentoalveolares & Postalveolar & Retrofleja & Palatales & Velares & Glotales \\
\hline Obstruyentes & $\mathrm{p}$ & $\mathrm{t}$ & $\underline{t}$ & & $\subseteq$ & $\mathrm{k}$ & $?$ \\
\hline Africadas & & $\mathrm{ts}$ & & & $\mathrm{t} \int$ & & \\
\hline Fricativas & & $\mathrm{s}$ & & $s$ & 6 & $\mathrm{x}$ & $h$ \\
\hline Sonorantes orales & $\beta$ & $\mathrm{r}$ & & & $\mathrm{j}$ & $\mathrm{u}$ & \\
\hline Nasales & $\mathrm{m}$ & $\mathrm{n}$ & & & $\mathrm{n}$ & $\eta$ & \\
\hline
\end{tabular}

El estatus fonológico de algunos de estos segmentos (representados con letra inclinada en la Tabla 1) se

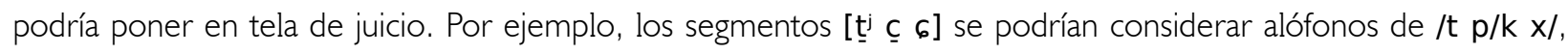
respectivamente, ya que hay amplia evidencia de que sus ocurrencias son consecuencia de la operación de un proceso regular de palatalización progresiva. Sin embargo, ese proceso antecede a un proceso no completamente regular de aféresis vocálica, lo que justifica la atribución de estatus fonológico a / El mismo raciocinio se aplica al segmento / $\mathbf{m / , ~ c u y a ~ o c u r r e n c i a ~ e s ~ p r e d e c i b l e ~ ( v e r ~ ' R e s o l u c i o ́ n ~ d e ~ h i a t o s ~ y ~ e s t a t u s ~}$ de $\eta, \boldsymbol{u}^{\prime}$ ) pero cuya inserción antecede al proceso morfofonológico de nasalización, justificando el reconocimiento del estatus fonológico de / $\mathbf{m} /$.

3 Uno de los estímulos para la adquisición de un nivel básico del bésiro por los alumnos es el hecho de que la competencia en la lengua originaria sea un requisito para la contratación en la Administración Pública del Estado Plurinacional de Bolivia.

4 Los datos en este artículo se representan en el Alfabeto Fonético Internacional (AFI), con la excepción del acento en las representaciones fonológicas, que se representa mediante la tilde ('). Utilizo los diacríticos de retracción en / $/ \underline{\mathrm{t}} \mathrm{c} /$ para subrayar que estos fonemas se articulan en las zonas postalveolar (= prepalatal) y pospalatal (= prevelar), respectivamente. El diacrítico de descenso en $/ \beta /$ indica que se trata de una aproximante y no de una fricativa. La africada /t $\int /$ se clasifica como [+ palatal] y no como [+ postalveolar] - - a pesar de articularse en la zona postalveolar — porque de otro modo sería necesario explicar por qué la consonante /ts/ no se palataliza a /t $5 /$ en el mismo ambiente en que /t/ se palataliza a /țj/ (ver 'Palatalización').

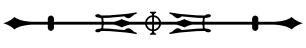


En (1-21), se demuestran algunos contrastes fonológicos con pares mínimos o análogos. Por razones de espacio, se dará preferencia a los pares de segmentos para los que se haya reportado ausencia de contraste en otras variedades del chiquitano ${ }^{5}$.

(1) $\quad / \beta / \times / m /:$

[çißi+ri'jix] 'su nervio'

[ßßа'kax] 'vaca'

[ßß口ato'kax] 'gualele'

[ta' $\boldsymbol{\beta a x}$ ] 'yuca'

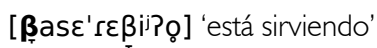

(2) $\quad / \mathrm{t} / \times / \mathrm{t} /$ :

(3) $\quad / \mathrm{ts} / \mathrm{x} / \mathrm{s} /$ :

(4) $/ \mathrm{s} / \times / \mathrm{s} /$ :

(5)

$|s| \times|6|:$

(6) $/ \mathrm{ts} / \times / \mathrm{t} / \mathrm{s}$ [tu'rux] 'turu'

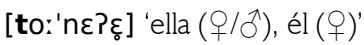

[taku'?̣ux] 'tacú'

[to' $\beta^{w}$ i:tso] 'está saltando'

[ti] 'ya', aspecto perfecto

[otsi'jọpip] 'me mordió'

[عtsimo' riçą] 'llevé huasca' [xaru'tsix] 'hormiguero (ㅇ)'

[tsaina'? ?ama?] 'cerquita'

[tsu' $\beta^{w}$ akatij] 'nos fuimos. EXCL'

[osto'nes] 'estrella (ठ̋')'

[suß ${ }^{w} \varepsilon^{\prime}$ rax] 'suela'

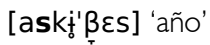

[عtșít'pơską] 'me casé'

[asto'płką] 'buenos días'

['saß̧arụ] 'sábado'

['soes] 'palo, árbol'

['Bis:i, ,o] 'es recto'

[kami'sax] 'camisa'

[sa'max] 'araña (†)'

[ki'tfutsaßo] 'se hinchó'

[tsa'ux] 'ayoreo (ㅇ)'

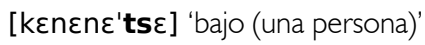

['tsarukị] 'mi labio'

[etsiri'piką] 'tengo hambre' [çi' mix ] 'gusano (P)'

[ma:'kax] 'viento sur'

[mato'rix] 'tarechi (P)'

[tama'?̧a] 'uno'

[ma'ses] 'masi (P)'

[țju'rux] 'puerta'

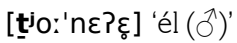

['țjakugta] 'porque'

[țjo' $\left.\beta^{w i} i\right]_{6}$ 'noche'

['țiji:] 'su cuello (de $X)$ '

[ose'jox] 'maíz'

[eșißָo'riçą] 'estoy viviendo'

[kuru'six] 'cruz'

['saíc] 'seis'

[sußw'a'ic] 'harto, gran cantidad'

[poştu'عs] 'portugués (idioma)'

[so $\left.\beta^{w} \varepsilon s^{\prime} t o x\right]$ 'Roberto'

[asko'res] 'caracoré'

[koski' rọ: rعg] 'corregidor'

[,jasta'Pti:mo] 'lo botó'

[6a'ßox] 'jabón'

[6o'? ع̃s] 'campo, pampa'

[taị.j't'pox] 'tijera (para cortar)'

[oßi'cax] 'oveja'

[Ga:'rax] 'cruceño (ठ')'

['ßutfaßo] 'bebieron (ㅇ)'

[tكaku'?̣ux] 'su espalda'

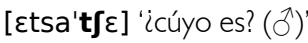

['t\}ariço] 'se peyó'

[çєmet fj' rix] 'cidrillo'

5 Las abreviaciones se enumeran al final del artículo. Para el significado de los términos regionales, ver 'Glosario'.

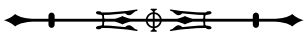


(7) $\quad / \mathrm{t}^{\mathrm{j}} / \mathrm{x} / \mathrm{t} / \mathrm{l}$

(8) $/ \underline{\mathrm{j} /} \times \mathrm{c} /$ :

(9)

/r/ x/n/:

(10)

$/ \mathrm{r} / \mathrm{X} / \mathrm{s} /:$

$(11)$

$/ \mathrm{n} / \mathrm{x} / \mathrm{n} / \mathrm{:}$

$/ \mathrm{j} / \mathrm{x} / \mathrm{n} /:$

(12) ['ițjąa] 'sobre él, en su cabeza'

[t'ja'kic] 'su piel, su cuero'

[țjape'?ux] 'su espalda'

[pi'țjux] 'pipa'

[oittji'mix] 'tigre $(\widehat{\jmath})$ '

['ițja] ] 'sobre él, en su cabeza'

[țja'?ic] 'su dureza'

[t'ja'kix] 'su tronco, su chala'

[țja'pax] 'su pierna'

[t'tu'sux] 'puerta'

[pit]a'rax] 'veneno'

[tsoko'res] 'socori (o)'

['ra:pic] 'lápiz'

['porre?o] 'está podrido'

[ caju'raką] 'estoy ayudando (ㅇ)'

[aş'porkax] 'alforja'

[karc'tax] 'carroza, carretón'

[osßar'tox] 'Osvaldo (o)'

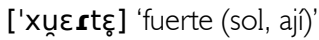

[li'mơrna] 'limosna'6

[mako'nox] 'frejol gateador'

[ono'nعs] 'tucán'

['pi:no] 'es lindo'

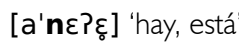

['nepi] 'eso si' ['ịititạ] 'mi rodilla'

[t]aku'??ux] 'su espalda'

[t]a'pox] 'vaso'

[kißi't\}ox] 'camote'

[tsoĩit fi'môkå] 'nos sentamos.INCL'

['iça] ] 'mi brazo'

[ça'?̦ic] 'su hueso'

[ça'kix] 'su madre de él (ㅇ)

[ca'pax] 'su madre de ella'

[cu'rox] 'letra'

[pitsa'nax] 'bolsa'

[tako'nعs] 'caña de azúcar'

[na:'kic] 'maní'

['pencko] 'lo botaron'

[Gatu:'naka] 'estoy nadando (o)'

[as'katع.] 'alcalde ()'

[kaşع'terax] 'carretera'

[soß $\left.{ }^{w} \varepsilon \mathbf{s}^{\prime} t o x\right]$ 'Roberto'

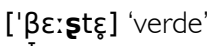

[as'mentrax] 'almendra'

[mako'nox] 'macono (q)'

[o'nes] 'jone'

['i:no] 'para mí ()'

[a'nes] 'carne'

[nعst'naką] 'está relampagueando'

[mako'nox] 'macono (q)'

[o'nعs] 'jone'

[notoxĩrjatą] 'lo colgó'

[sano'rax] 'señora'

[anc'tux] 'su carnaza'

6 En esta propuesta, /I/ no se considera parte del inventario fonológico del chiquitano migueleño; [I] ocurre en algunos ítems en el

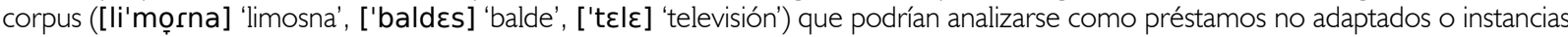
de alternancia de códigos (code-switching). Alternativamente, /l/ podría considerarse un fonema marginal.

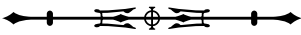


(13)
$/ \mathrm{p} / \mathrm{x} / \mathrm{c} /:$
[pi'tsic] 'negro, moreno (P)'
['pơ: x] 'casa'
[pa'tax] 'alacrán (q)'
[paxpa'kic] 'sucha (\%)'
[ras' pą̧rax] 'San Rafael'

(14) $/ \mathrm{k} / \times / \mathrm{c} /$ :

(15)

$|x / x / 5|:$

$\mid x / x / 2 /:$

(16)

(17)

(18) [ka: 'sikị] 'cacique (†)'

[kopi'?̦i: roo] 'está jugando'

[ki'ric] 'lagarto (ㅇ)'

[kaxka' røs] 'cascaré (o)'

['pikox] 'pico (beso)'

[a'xi6] 'jachi'

['axtaka?] 'hasta que'

['piìxtą] 'fiesta, cumpleaños'

[kaxka'res] 'cascaré (o)'

['tre: $\mathbf{x}]$ 'tres'

['axpcku?] 'entre'

[xorijo'kox] 'soriocó'

[kaxka'res] 'cascaré (o)'

[kaxtri'janux] 'castellano'

['axtaka?] 'hasta que'

[xa:' rax] 'cruceño (ㅇ)'

[xorijo'kox] 'soriocó'

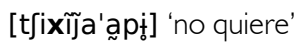

[xi'cọx] 'ratón (P)'

[xuß̣ ${ }^{w} \varepsilon^{\prime}$ jọką] 'está lloviznando'

[xa:' rax] 'cruceño (ㅇ)'

[xorijo'kơx] 'soriocó'

[xiripi'tix] 'trapo'

[xi'cọx] 'ratón (官'

[xa'?̦í6] 'puchi, excrementos' [çi'tsí] 'negro, moreno (ふ̋)'

['coo:x] 'su casa'

[ça:'tax] 'otro'

[naxça'?ִ̣̂na?] 'su gajo'

[josçaki'jox] 'paquió'

[ça: 'sikị] 'cacique (đ̂)'

[co'prs] 'su pie'

[ćc't'tsic] 'negro, moreno (ふ̋)'

[naxça'tax] 'su flojera'

['ico] ] 'mi casa'

[a'sig] 'curupaú'

[asto'płka] 'buenos días'

[osto'nes] 'estrella (ふ̋)'

[aski'ß̦عs] 'año'

['sọes] 'palo, árbol'

[as' porkax] 'alforja'

[soß̦ $\left.{ }^{w} \varepsilon s^{\prime} t o x\right]$ 'Roberto'

[aşko'res] 'caracoré'

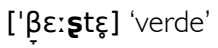

[.jasta'Ti:mo] 'lo botó'

[Ga:' rax] 'cruceño (ô)'

[6oriça'kiø] 'su bofe; esp. de sepe'

[iడ̄ĩjakąa] 'quiero'

[డĩ̃jes] 'anguila (o)'

[6o'ux] 'víbora (क)'

['(h)ap $]_{\text {] }}$ 'arriba'

[(h)o'nع] adverbio de pasado

['(h)im] 'a ella (\%/ठ̋), a él (\%)'

[(h)iit'rotit] 'que yo vaya'

[(h)a' $\varepsilon m$ ] 'a vos'

7 El estatus fonológico de /h/ es problemático, como se discutirá en la sección 'Estatus de $h$ '. Un(a) evaluador(a) anónimo propone atribuir las ocurrencias de /h/ que alternan con cero a una regla de epéntesis; en este caso los ejemplos en (18) ilustrarían el contraste entre / $\mathbf{x}$ / y cero.

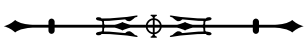




$$
/ m / x / \beta /:
$$

$/ m / x / y /:$

$|\beta / \times| \varnothing \mid:$

$$
\begin{aligned}
& \text { ['sti: } \mathbf{y o u}_{\text {o. }} \text { 'está seco (planta)' }
\end{aligned}
$$

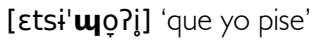

$$
\begin{aligned}
& \text { [ki' 'щơrii?o] 'llevó huasca' } \\
& \text { [kima'rax] 'azucaró (o)' } \\
& \text { [i' 'uyox] 'urina (\%)' }
\end{aligned}
$$

$$
\begin{aligned}
& \text { [ki'mox] 'urucú' } \\
& \text { [kiłmox'kiø] 'pala' }
\end{aligned}
$$

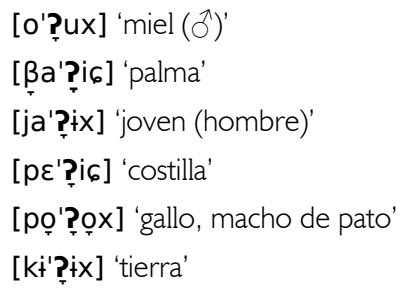

['Kijißฺo] 'nació'

[عtsit' $\beta$ ợ: $\beta i]$ ] 'en mi barriga'

[si' $\beta$ orriço] 'está viviendo'

[arißß又a'?̦akax] 'albahaca'

[aski' $\boldsymbol{\beta} \varepsilon s]$ 'año'

\section{[ki'noma?] 'urucucito' \\ [kinox'kĩ̄a?] 'palita'}

\author{
[o'ux] 'paja' \\ [ß̧a'i६] 'curichi' \\ [ja'ix] 'su padre de él (P)' \\ [pe'i i ] 'peji (ㅇ)' \\ ['po:x] 'casa' \\ ['ki:x] 'capiguara, cujuchi (P)'
}

La realización fonética de los fonemas consonánticos será discutida en la próxima subsección, mientras que en las subsecciones subsiguientes se proporcionará la evidencia que podría utilizarse para cuestionar el estatus fonológico de los segmentos /țj $\subseteq 6$ s h u $\mathrm{\eta} /$.

\section{REALIZACIÓN FONÉTICA DE LAS CONSONANTES}

La realización de los segmentos /p $\mathrm{m} \mathrm{t} \mathrm{n} \mathrm{n} \mathrm{t} 6 \mathrm{j} \mathrm{k}$ u $\mathrm{g} /$ es invariable y corresponde a sus valores en el Alfabeto Fonético Internacional (AFI) ${ }^{8}$. Obsérvese que en esta propuesta ciertas alternancias entre consonantes (como aquellas que involucran la palatalización) se atribuyen a procesos que operan en el nivel morfofonológico, como se mostrará en las secciones subsiguientes. Por ello, no se considera, por ejemplo, que el fonema /t/ posee un alófono [țj], sino que se propone postular una alternancia morfofonológica entre los fonemas /t/ y / t $\mathrm{t} /$ ( $\mathrm{y}$ otros pares similares).

El segmento / $\boldsymbol{\beta} /$ normalmente se realiza como una aproximante bilabial $[\boldsymbol{\beta}]$, aunque en algunos casos su realización puede oscilar. Más específicamente, en el comienzo de las palabras se ha registrado la ocurrencia opcional del alófono oclusivo [b], posiblemente por la interferencia del castellano. Además, tras las vocales /o, u, $\dot{t}$ / el fonema / $\beta$ / frecuentemente muestra un efecto coarticulatorio (muy similar a lo que ocurre en el español cubano) que consiste en el mantenimiento del redondeamiento labial durante toda la duración de la aproximante, resultando en las realizaciones como $\left[\beta^{w}\right],[w]$ o incluso $[\varnothing]$ (en este último caso la vocal precedente puede reducirse a $\left.[w]\right)^{9}$. Algunos ejemplos se dan en (22).

\footnotetext{
8 Un análisis auditivo sugiere que el sonido que se representa aquí como [t] es una oclusiva dental y no alveolar, hecho que debería representarse en el AFI mediante la utilización de un diacrítico dental ([t]]). Sin embargo, no se ha podido realizar un análisis palatográfico que podría comprobar esta impresión auditiva. Por esta razón, en este artículo se mantendrá la utilización del símbolo [t].

9 No está completamente claro por qué la vocal no redondeada /ł/ desencadenaría tal realización.
} 


\begin{tabular}{|c|c|c|c|}
\hline \multirow[t]{4}{*}{ a. } & /ßֵuxí́i// & {$\left[\beta u^{\prime} x i 6\right]$} & 'tigre (o)' \\
\hline & /ß̦akíci/ & [ß̧a'kic] & 'garza (Q)' \\
\hline & /ß̦ístro/ & {$[' \beta i s: \underset{t}{i}, 10]$} & 'es recto' \\
\hline & /6aßฺóxit/ & [6a'ßָox] & ‘jabón’ \\
\hline \multirow[t]{2}{*}{ b. } & 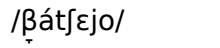 & 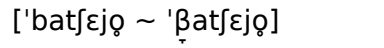 & ‘dio’ \\
\hline & /ßָáPa poókaa/ & [,ba:: 'po:ka , ßָa: 'po:ka] & 'las casas' \\
\hline \multirow[t]{4}{*}{ c. } & /kuß̧áti/ & [ku' $\beta^{w}$ atị $\sim$ 'kwatị ] & 'viene' \\
\hline & /عtsuß̄uxíça/ & [عtsuwu'xiçąa عtsu:'xiçą] & 'me tapé' \\
\hline & /عtsoßָoríça/ & [عtsowo' riça عtso: 'riça] ] & 'me preparé' \\
\hline & /tißßatsíxit/ & [tiß $\beta^{w}$ a'tsix $\sim$ tiwa'tsix] & 'pato (P)' \\
\hline
\end{tabular}

A pesar de que los segmentos / $\mathbf{t} \mathbf{j} /$ y /ç/ posean una realización constante (salvo en la pronunciación de los semihablantes, que suelen realizarlos como [t] y [ki], respectivamente), es oportuno discutirla aquí en más detalle, ya que los fonos que les corresponden son similares acústica y perceptualmente. El segmento /țí/ se realiza como una oclusiva sorda, cuyo punto de articulación es posalveolar (o prepalatal); la configuración de la lengua durante la obstrucción de este fono puede describirse como laminal o antedorsal (algo que no se ha podido averiguar palatográficamente). Por falta de un símbolo dedicado del Alfabeto Fonético Internacional, este fonema y su fono correspondiente se representarán

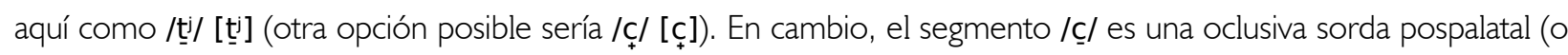
prevelar), articulada con la zona antedorsal de la lengua. Este fonema y su fono correspondiente podrían representarse

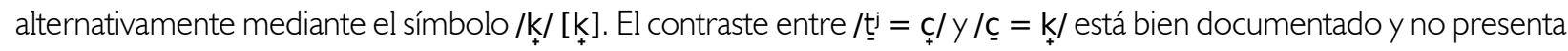
ninguna dificultad para los hablantes del chiquitano migueleño.

Los segmentos /ts/ y /s/ se realizan siempre como [ts] y [s], respectivamente, en el habla tanto de una de las consultantes del autor en San Juan de Lomerío como de un consultante originario de San Pedro de Sapocó (cuyos datos no fueron analizados en este artículo). Un consultante originario de San Miguel de Velasco muestra una fuerte tendencia a realizar los dos segmentos como [s]. Finalmente, los idiolectos de otras consultantes presentan algunas particularidades que podrían estar o no relacionadas con el estado deteriorado de su dentición. Una consultante de San Juan no reproduce la distinción entre /ts/ y /s/ de forma sistemática, a veces realizando /s/ como [ts] y, con una frecuencia menor, /ts/ como [s] (esta misma hablante a veces pronuncia /tf/ como [ts]). Otra consultante, de San Pedro de Sapocó, mantiene la distinción entre /s/ y /ts/, pero realiza este último segmento como una oclusiva dental [t] (no se analizarán sus datos en este artículo).

El segmento /s/ se realiza con un grado variable de fricción: [s $\sim$ ș ז̊], igual a su equivalente en el castellano camba.

El segmento /n/ suele realizarse como una oclusiva nasal palatal sonora [n] (23a), pero tras la vocal anterior alta /n/ puede perder la oclusión y se realiza como una aproximante nasalizada, nasalizando también la vocal precedente (23b).

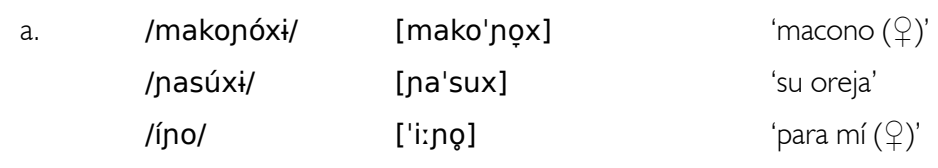




\begin{tabular}{|c|c|c|}
\hline /ináxit/ & [ĩ̃ax] & 'su nariz' \\
\hline /inásu/ & [ĩ̃jasụ] & 'mi oreja' \\
\hline /pinóoniçi/ & [pĩץō̃̃:niG] & 'piñón' \\
\hline /inatápi/ & [ĩ̃a'ta?ị ina'ta?ị] & 'llegó' \\
\hline /pinźsł̇/ & [pĩ̃jes] & 'peni, jaúsi (Q)' \\
\hline
\end{tabular}

La realización del segmento /x/ parece oscilar entre $[\mathrm{x}]$ y [h] tanto en los ataques como en las codas (24). No se ha detectado ninguna ocurrencia de [h] en la posición final de la palabra.

\begin{tabular}{|c|c|c|c|}
\hline \multirow[t]{2}{*}{ a. } & /xarutsíxit/ & [xaru'tsix $\sim$ haru'tsix] & 'hormiguero (O)' \\
\hline & /kuxurźsł̇/ & 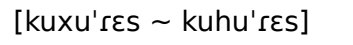 & 'piraña (q)' \\
\hline \multirow[t]{2}{*}{ b. } & /axtsaríci/ & [axtsa'?ৃic ahtsa'?ָi६] & 'jasayé' \\
\hline & /paxpakíci/ & [рахра'kiє pahpa'kiє] & 'sucha (Q)' \\
\hline
\end{tabular}

En cambio, apenas un alófono, [h], ha sido atestiguado para el supuesto segmento /h/, de muy baja frecuencia en el corpus (ver subsección 'Estatus de $h$ ' para algunos ejemplos).

Finalmente, el segmento / $/$ / raramente se realiza como una verdadera oclusiva glotal en chiquitano migueleño. La oclusión completa suele conservarse precediendo una vocal ensordecida o en posición final, que sólo resulta de elisión de la vocal final (ver 'Ensordecimiento y elisión') (25a). En cambio, en la posición entre dos vocales no ensordecidas / P/ prototípicamente pierde la oclusión, realizándose como una aproximante [?] o incluso como una fonación no modal (voz tensa o voz crujiente ${ }^{10}$ ) en la vocal siguiente $(25 b, c)$, tal como sucede en un sin número de lenguas del mundo (cf. Ladefoged \& Maddieson, 1996, p. 75). Esta última posibilidad es particularmente frecuente cuando / $/$ / se encuentra entre vocales átonas (25c).

\begin{tabular}{|c|c|c|c|c|}
\hline \multirow{3}{*}{\multicolumn{2}{|c|}{ (25) }} & /çipópi/ & [çi'popị] & 'está pisando' \\
\hline & & /ÍțoPo/ & ['ițoP] & 'también' \\
\hline & & /nac̃támaPa/ & 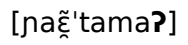 & 'solito (ఏ)' \\
\hline & \multirow[t]{3}{*}{ b. } & /sapáxì/ & [sa'Paax] & 'paja sujo (O)' \\
\hline & & /part́xt/ & [pa'P̣ix] & 'mujer' \\
\hline & & /60PẼst/ & 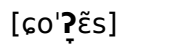 & 'campo, pampa' \\
\hline & \multirow[t]{3}{*}{ c. } & /aß̧ع́?દko/ & [a'ßָعદ్లko] & 'está satisfecho' \\
\hline & & /kóta?a/ & ['kotaą] & ‘idónde?' \\
\hline & & /tsáPa poóxì/ & [,tsa : 'po:x] & 'esta casa' \\
\hline
\end{tabular}

\footnotetext{
10 Aquí no se hará un intento de distinguir entre esas fonaciones y se utilizará la notación [V] para representar cualquier tipo de vocales no modales del chiquitano migueleño. Obsérvese que algunos autores emplean los términos 'voz tensa' y 'voz crujiente' refiriéndose a dos grados distintos de constricción de la glotis, que pertenecerían a un mismo continuo entre la fonación modal y el estado totalmente constreñido de la glotis. Otros autores definen la voz crujiente (creak en inglés) como una fonación irregular, sin la implicación de que las cuerdas vocales estén más constreñidas a comparación de la voz modal. Futuros estudios deberán determinar cómo exactamente se producen las vocales denotadas aquí como [V].
} 


\section{PALATALIZACIÓN}

La palatalización progresiva es un proceso omnipresente en el chiquitano migueleño; consiste en que las consonantes

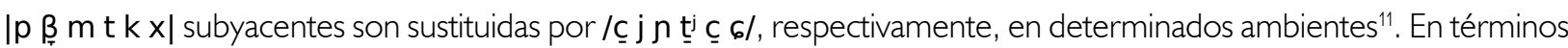
de los rasgos distintivos, se podría proponer la siguiente generalización: las consonantes [+labiales] y [+velares] se palatalizan a [+ palatales] manteniendo el modo de articulación, mientras que la consonante [+dentoalveolar] |t| se palataliza a su contraparte [+ postalveolar] /țj/ (las demás consonantes [+ dentoalveolares] —esto es, |ts s r n|— no se palatalizan, ya que el inventario del chiquitano migueleño no tiene africadas, fricativas o sonorantes [+ postalveolares]). Para las consonantes $|\mathrm{p} \beta \mathrm{m} \mathrm{t} \mathrm{k}|$, el ambiente condicionante es la posición entre una vocal |i| o |ĩ| subyacente y cualquier vocal que no sea $\mid i$ i $\left.\right|^{12}$. Esta última condición no se aplica a la $|x|$, que se palataliza tras $\mid i$ i $\mid$ sin importar la vocal que le sigue. Las evidencias que muestran que la palatalización es un proceso activo incluyen los siguientes hechos:

i. hay numerosos ejemplos de alternancias entre $/ \mathrm{p} \underset{\beta}{\beta} \mathrm{m} \mathrm{t} \mathrm{kx/y/ \subseteq} \mathrm{j} \mathrm{n} \underline{\mathrm{t}} \underline{\mathrm{c}} \mathrm{6} /$;

ii. $/ \mathrm{p} \beta \mathrm{m} \mathrm{t} \mathrm{k} \mathrm{x/} \mathrm{no} \mathrm{ocurren} \mathrm{en} \mathrm{el} \mathrm{ambiente} \mathrm{/i...V/} \mathrm{O} \mathrm{/ĩ...V/} \mathrm{(salvo} \mathrm{si} \mathrm{V}=\mathrm{i} / \tilde{\mathrm{i}}$ en el caso de las primeras cinco consonantes), con contadísimas excepciones;

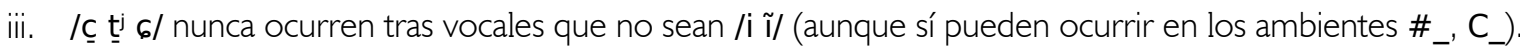

Hay dos dificultades posibles para el análisis propuesto aquí. La primera consiste en la existencia de palabras en las que la palatalización no ocurre a pesar de que haya condiciones para ello. Se trata de algunos préstamos del castellano y, en el subdialecto de San Juan, de las formas que contienen el prefijo negativo/condicional /t $\mathrm{t} \mathrm{i}-/$ (en San Miguel y en otras comunidades este mismo prefijo tiene la forma /t $\left.\int \mathrm{i}-/\right)$, como se muestra en (26).

\begin{tabular}{|c|c|c|c|}
\hline /ríkoxit/ & ['rikox] & 'rico' & $<$ cast. rico \\
\hline /píkoxit/ & ['pikox] & 'beso' & $<$ cast. pico \\
\hline /sĩku/ & ['sĩnku] & 'cinco' & $<$ cast. cinco \\
\hline /anitáxí/ & [ani'tax] & 'Ana' & $<$ cast. Anita \\
\hline 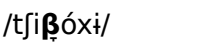 & [t]i' $\boldsymbol{\beta} O \boldsymbol{T} \mathrm{x}]$ & 'chivo' & $<$ cast. chivo \\
\hline /rímaxit/ & ['rimax] & 'lima' & $<$ cast. lima \\
\hline /rasímoxit/ & [ra'simox] & 'racimo' & $<$ cast. racimo \\
\hline /tكipakoáijopí/ & [t]ipa'koaijopị] & 'no es ancho' & (San Miguel: /t $\int \mathrm{i}-/\left[\mathrm{t} \int \mathrm{i}-\right]$ ) \\
\hline /tكixina?ápi̇/ & 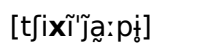 & 'no quiere' & (San Miguel: /t $\int \mathrm{i}-/\left[\mathrm{t} \int \dot{\mathrm{i}}-\right]$ ) \\
\hline
\end{tabular}

Sin embargo, en la mayoría de los préstamos las secuencias 'ilíitas' se eliminan por el proceso de palatalización, como se muestra en (27) a continuación.

11 Las representaciones morfofonológicas (subyacentes) se dan entre plecas (| . . $\mid$ ), mientras que las barras oblicuas $(/ \ldots /)$ se reservan para las representaciones fonológicas. Los archifonemas se representan con una notación fraccionaria, con una posibilidad indicada en el numerador y otra en el denominador (como, por ejemplo, cuando no se puede determinar si una ocurrencia de /ç/ es derivada de $|\mathrm{p}|$ o de $|\mathrm{k}|$ ). La morfofonología del chiquitano se discute de un trabajo aparte (Nikulin, 2020b).

12 La vocal |ĩ| es de muy baja frecuencia en el chiquitano migueleño, por lo que las instancias de palatalización que involucran esta vocal

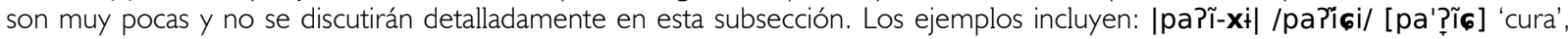

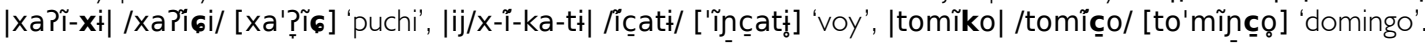

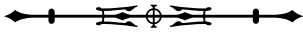


(27)

\begin{tabular}{|c|c|c|c|}
\hline /miçoráxit/ & [miço'rax] & 'Nicolás' & < cast. Nicolás \\
\hline /tomĩço/ & [to'mĩñcoo] & 'domingo' & $<$ cast. domingo \\
\hline /ßָuriçáxí/ & [ßßuri'çax] & 'mula' & $<$ cast. burrica \\
\hline /íkkareráxí/ & [iøkars'rax] & 'escalera' & $<$ cast. escalera \\
\hline /maíctiru/ & [ma'ictí, ru] & 'profesor (P)' & $<$ cast. maestro \\
\hline /iøpinițúmara/ & [iєpini'țuma?] & 'picaflor' & $<$ cast. espíritu + DIM \\
\hline
\end{tabular}

La segunda dificultad consiste en el hecho de que la vocal que desencadena la palatalización puede sufrir aféresis (en la posición inicial) o síncopa (en posición átona entre dos consonantes sordas). Algunos ejemplos se dan en (28).

\begin{tabular}{|c|c|c|c|}
\hline$\left|i_{1}-\operatorname{taPani}-x \dot{x}\right|^{13}$ & /țảanáxít/ & [țja: & 'su cabeza' \\
\hline $\mid i_{1}-\beta$ - & /jotóxt/ & [jo'tọx] & 'su nalga' \\
\hline |i $i_{2}$-penc-ro-nipi| & /çعnعnóniłił/ & 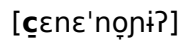 & 'me botó, me empujó' \\
\hline $\mid i_{j}^{\frac{x}{j}-i_{2}}$-tasu-ka-ipi & /țjasúkiłił/ & [țja'sukipi?] & 'te llamé' \\
\hline |ixou-xi| & /6oúxí/ & [60'ux] & 'víbora (q)' \\
\hline $\mid i \frac{p}{k}$ orera-xit| & /çoreráxìt/ & [core'rax] & 'corredor' \\
\hline |i $i_{2}-k o s \varepsilon \varepsilon-x i \mid$ & /çoseźst'/ & [ço'se:s] & ‘José (ふ઼)' \\
\hline$\left|i_{2}-k a a r u x i-x+1\right|$ & /çaaruxí́ii/ & [ça:ru'xí] & 'Carlos (ठ̋')' \\
\hline $\mid j o r i \frac{p}{k}$ aki $\frac{\beta}{j} 0-x+\mid$ & /joscçakijóxit/ & [josccaki'jơx] & 'paquió' \\
\hline $\mid i_{1}-\max i_{k}^{\frac{p}{k}}$ ata- $x \dot{i} \mid$ & /naxçatáxił/ & [naxça'tax] & 'su flojera' \\
\hline
\end{tabular}

Al contrario de lo que ocurre en lenguas como el kari'ña (familia caribe) (ver Álvarez, 2016, pp. 23-24), que también presenta un proceso de palatalización progresiva seguido de un proceso de aféresis vocálica, en chiquitano migueleño la aféresis de |i| no es un proceso estrictamente fonológico, ya que es imposible predecir su aplicación sin

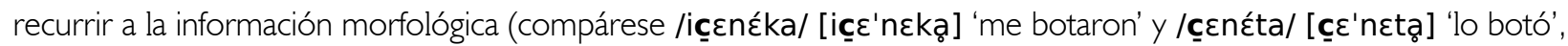
con prefijos distintos). Por esta razón, la palatalización debe atribuirse al nivel morfofonológico.

A continuación, se ilustra la aplicación regular del proceso de palatalización progresiva desencadenado por los

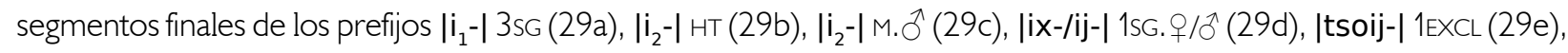
así como por la vocal final de las raíces (29f), afectando los segmentos iniciales de los sufijos |-xi| X, |-kaa| PL, |-maPa| DIM, |-ka| F.SAP, |-ko| F.3, |-ka| F.CLIM, |-ta| 3sG.oB], |-taiki| PL.KIN, |-po| NMLZ.INSTR, |-ßָo| TRANSL.

$$
\text { Palatalización progresiva }
$$

a.

$$
\left|i_{1}-\right| \text { 3sG: }
$$

\begin{tabular}{|c|c|c|c|}
\hline & & $|p| \rightarrow / c \mid$ & \\
\hline /içáxit/ & [i'ćax] & 'su brazo' & cf. 2sG /ápa/ ['apą] \\
\hline /çعદkíci// & 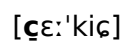 & 'su fiebre' & cf. 2sG /apézki// [a'pz:kị] \\
\hline /çopésł'/ & [co'pes] & 'su pie’' & cf. 2sG /ápope/ ['ароpє̨] \\
\hline /çitáxit/ & [ci'tax] & ‘su talón' & cf. 2sG /apîta/ [a'pitå] \\
\hline
\end{tabular}

\footnotetext{
${ }^{13}\left|i_{1}\right|$ denota una variante de $|\mathrm{i}|$ que se elide delante de vocales, mientras que $\left|\mathrm{i}_{2}\right|$ denota una variante de $|\mathrm{i}|$ que se transforma en $/ \mathrm{j} / \mathrm{o}$
} /n/ en el mismo ambiente.

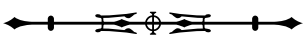




\begin{tabular}{|c|c|c|c|}
\hline & & $|\mathrm{t}| \rightarrow / \mathrm{t}^{\mathrm{j}} /$ & \\
\hline /țjaPaníxít/ & [țjad:'nix] & 'su cabeza' & cf. 2sG /atá?aní/ [a'taąṇ] \\
\hline /țjokirãxit/ & [țjokij'?ãx] & 'su ombligo' & cf. 2sG /atókirã/ [a'tọkii?å̃] \\
\hline 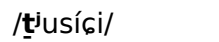 & [țju'sig] & 'su pecho' & cf. 2sG /átusi// ['atusị] \\
\hline$/ \underline{\mathbf{t}} \dot{\mathbf{t}} \dot{\mathrm{t}} \times \dot{x} /$ & {$\left[\underline{t}^{\mathrm{j} j \mathrm{i}} ; \mathrm{x}\right]$} & ‘su cuello' & cf. 2sG /átïï/ ['atti] \\
\hline & & $|\mathrm{k}| \rightarrow / \mathrm{c} /$ & \\
\hline /çعmésł'/ & [ç $\left.\varepsilon^{\prime} m \varepsilon s\right]$ & 'su espina' & 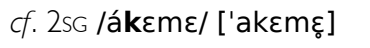 \\
\hline /çomésł́/ & [co'mes] & 'su escopeta' & cf. 2sG /ákome/ ['akomę] \\
\hline /ćipoositoxitíri/ & [ćcipo:stox'ti?] & 'su esposo (đ̋')' & cf. 2sG /akipóosił/ [akị'pơ:s] \\
\hline & & $|\beta| \rightarrow|\rightarrow j|$ & \\
\hline /jaitsí́i// & [jaj'tsí ] & 'su hamaca' & cf. 2sG /aß口áitsi/ [a'ß]aitsi] \\
\hline /jo?óxt/ & [jo'?ִơx] & 'su chaco' & 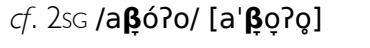 \\
\hline & & $|\mathrm{m}| \rightarrow / \mathrm{n} /$ & \\
\hline /nomésł́/ & [no'mes] & 'su chipa' & cf. 2sG /amóme/ [a'mọ:mę] \\
\hline
\end{tabular}

b. $\quad\left|\mathrm{i}_{2}-\right|+\mathrm{HT}$

/çعnćta/

[çع'nztą]

$|\mathrm{p}| \rightarrow / \mathrm{c} /$

'lo botó

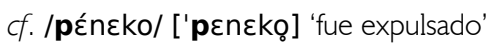

$|\mathrm{t}| \rightarrow / \mathrm{t} /$

/țiaßáaița/

[țja' $\beta$ aittiąa]

'lo mató'

[țjo'moztą]

'lo ató'

/țuméta/

[țju'metą ]

'lo ahorcó'

/țjimokóta/

[țijmo'kotą)

'lo asentó'

$|\mathrm{k}| \rightarrow / \mathrm{c} /$

/çáta/

['çatą]

/çonokóta/

[cono'kótą]

'lo llevó/sacó'

cf. /makáana/ [ma'ka:ṇ] 'llevó/sacó'

/çupúta/

[çu'putą]

'lo adornó'

cf. /akónoko/ [a'kơnoko] 'tu hermosura'

'lo mandó'

/çiuarítia/

[çima'ițią)

cf. /ßakúpuro/ [ßßа'kupuro] 'mandó'

cf. /ßָakiuyáiro/ [ßָaki'mairro] 'azotó'

c. $\quad\left|i_{2}-\right| M \cdot O^{\lambda}$ :

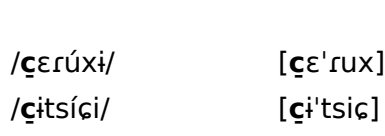

$|\mathrm{p}| \rightarrow / \mathrm{c} /$

'Pedro (đ̋')'

'negro (ð゚')'

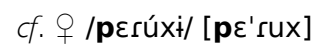

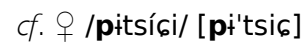

/țianiére/

[ța'niz:

$|\mathrm{t}| \rightarrow / \mathrm{t} /$

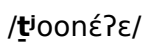

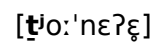

'Daniel $(\widehat{\jmath})$ '

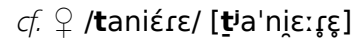

/țiupáxí/

[țu'pax]

'él $(\widehat{\zeta})$ '

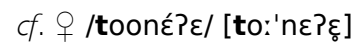

'Dios $(\widehat{\partial})$ '

cf. + /tupáxł// [tu'pax] 


\begin{tabular}{|c|c|c|c|}
\hline /çaasíki/ & [ça:'sikị] & $\begin{array}{l}|\mathrm{k}| \rightarrow / \mathrm{c} / \\
\text { 'cacique }(\widehat{\widehat{O}}) \text { ' }\end{array}$ & cf. + + /kaasíki/ [ka:'sikị] \\
\hline /çعsúuxì/ & [çs'su:x] & 'Jesús (ठํ)’ & cf. + / /kesúuxit/ [ke'su:x] \\
\hline /çosécsìt/ & [co'se:s] & 'José (ふึ)' & 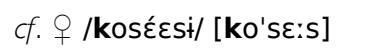 \\
\hline /çuxtíne/ & [çux'ti:ng̨] & 'Agustín (ठ)' & cf. + /kuxtíne/ [kux'ti:ng̨] \\
\hline $\begin{array}{l}\text { /naíctiru/ } \\
\text { /nźsku/ }\end{array}$ & $\begin{array}{l}\text { [na'iistit, ru] } \\
\text { ['neşkụ] }\end{array}$ & 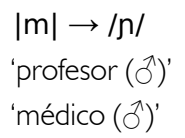 & 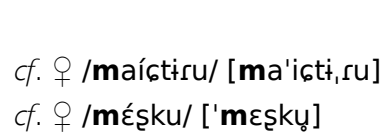 \\
\hline /Gaaráxí/ & [6a:'ráx] & $\begin{array}{l}|x| \rightarrow|6| \\
\text { 'cruceño }\left(\partial^{\lambda}\right)^{\prime}\end{array}$ & cf. ㅇ /xaaráxí// [xa:'ráx] \\
\hline
\end{tabular}

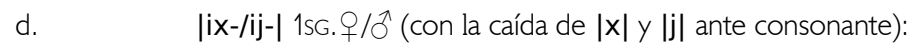

\begin{tabular}{|c|c|c|c|}
\hline & & $|\mathrm{p}| \rightarrow / \mathrm{c} /$ & \\
\hline liçakíça/ & [iça'kiçą] & 'me caí' & cf. 3sG /pákißo/ ['pakii?o] \\
\hline /içદnźka/ & [icç'ncką] & 'me botaron' & 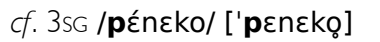 \\
\hline /içosíça/ & [iço'siçą] & 'soñé' & cf. 3sG /pósiço/ ['posiço] \\
\hline /iç̣̂ta/ & [i'ćctą & 'mi talón’ & cf. 2sG /apíta/ [a'pita] \\
\hline & & $|\beta| \rightarrow / j \mid$ & \\
\hline /ijáitsi/ & [i'jaintsi] & 'mi hamaca' & cf. 2sG /aßßáitsi/ [a'ß]aittsi] \\
\hline /ijópo/ & [i'jơ?o] & 'mi chaco' & cf. 2sG /aßָó?o/ [a'ßָô?o] \\
\hline & & $|\mathrm{m}| \rightarrow / \mathrm{n} /$ & \\
\hline /inásu/ & [î'j̃asụ] & ‘mi oreja' & cf. 2sG /amásu/ [a'masụ] \\
\hline /inóme/ & [ĩ'j̃o:mع̨] & 'mi chipa' & cf. 2sG /amóms/ [a'mo:mę] \\
\hline /icináka/ & [iøî̃jaką] & $\begin{array}{l}|x| \rightarrow|\varsigma| \\
\text { 'quiero' }\end{array}$ & cf. 2sG /axináka/ [axĩ̃jaką] \\
\hline
\end{tabular}

e. $\quad$ tsoij-| 1EXCL:

\begin{tabular}{|c|c|}
\hline & [tsoi'capu] \\
\hline
\end{tabular}

f. raíces que terminan en |i|:

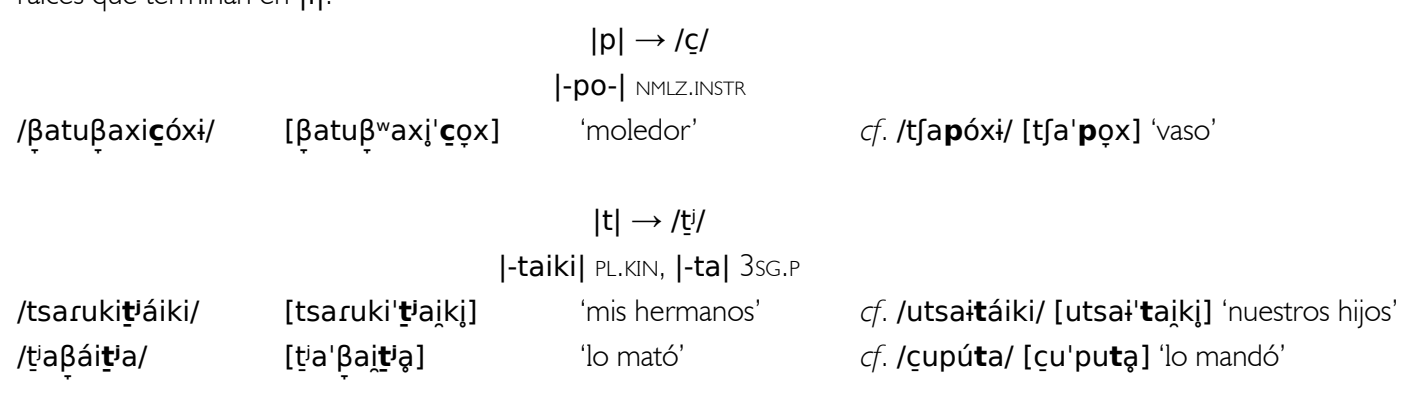




\begin{tabular}{|c|c|c|c|}
\hline \multicolumn{4}{|c|}{$|\mathrm{k}| \rightarrow / \mathrm{c} /$} \\
\hline /tfikíçaa/ & [t]i'kiça] & 'huevos' & cf. /tamokókaa/ [tamo'kôka] 'perros' \\
\hline /içakíça/ & [iça'kiçą] & 'me caí' & cf. /itfimóka/ [itfi'mōką] 'estoy sentado' \\
\hline /tosíßָiço/ & [to'sißßiço] & 'está gritando' & cf. /arćjoko/ [a'rعjoko] 'está llorando' \\
\hline /țjoß̦íça/ & [țo'wiça] & 'anochece' & cf. /taPáka/ [ta'?̣aka] 'llueve' \\
\hline & & 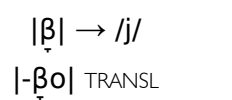 & \\
\hline /it]apikíjo/ & [it]api'ki:jo] & $\begin{array}{l}\text { 'para mi tapeque' } \\
|\mathrm{m}| \rightarrow / \mathrm{n} / \\
\text { |-maPa| DIM }\end{array}$ & cf. /içooßßo/ [i'ço: \\
\hline /anína?a/ & [a'nĩja?] & 'ajicito' & cf. /kapémaPa/ [ka'pema?] 'cafecito' \\
\hline & & $\begin{array}{c}|x| \rightarrow|6| \\
|-x \dot{\mid}| \times\end{array}$ & \\
\hline /t Jikíci// & {$\left[\mathrm{t} \int \mathrm{i}\right.$ 'ki6] } & 'huevo' & cf. /kaPaxí/ [ka'?ָax] 'piedra' \\
\hline
\end{tabular}

Los ejemplos en (30) muestran que las consonantes $\mid \mathrm{p} \mathrm{t} \mathrm{k} \beta \mathrm{m}$ | no se palatalizan cuando son seguidas de /i/. El bloqueo de palatalización progresiva en condiciones similares ha sido atestiguado en lenguas tales como el paresí (familia arahuaca) (ver Brandão, 2014, pp. 42, 46) y el ye'kwana (caribe) (ver Cáceres, 2011, p. 74), pero no en las demás variedades del chiquitano ${ }^{14}$.

\begin{tabular}{|c|c|c|c|}
\hline |tipi-xi| & /tipíçi/ & [ti'pic] & 'hormiga (P)' \\
\hline$\left|\mathrm{i}_{j}^{\frac{x}{j}}-\mathbf{p i i x} x t a\right|$ & /ipí́xta/ & [i'pïixta] & 'mi cumpleaños' \\
\hline $\mid \mathrm{i}_{j}^{\frac{x}{j}}$-pitu| & /ípițu/ & ['ipițịu] & 'mi pipa' \\
\hline$\left|i_{2}-p i r i p i\right|$ & /pirípi/ & [pi'ripị] & 'Felipe $(\lesssim)^{\prime}$ \\
\hline |tapiti-xí| & /tapitíci/ & [tapi'tic] & 'tapití (ㅇ)' \\
\hline |oi-ti?i| & /oitíîi/ & [oí'tî] & 'por él (ठ̋)' \\
\hline$\left|i_{1}-\mathbf{k i}-x \dot{t}\right|$ & /ikíci// & [i'ki६] & 'su pluma, su vello' \\
\hline |kupiki-xí| & /kupikíci/ & [kupi'kiø] & 'joven (mujer)' \\
\hline |kituriki| & /kitúriki/ & {$[$ ku'turi,ki] } & 'rojo' \\
\hline |kaasiki| & /kaasíki/ & [ka:'sikị] & 'cacique (q)' \\
\hline |ix-otuxiki| & /6otúxiki/ & [60'tuxịkijo] & 'mi saliva (q)' \\
\hline $\mid \mathbf{i}_{j}^{\underline{x}}$-tapiki $\mid$ & /itكapíki/ & [it]a'piki]] & 'mi tapeque' \\
\hline |aPißָi-xí| & /apißָíci// & [ap̣i'ßקic] & 'su ropa' \\
\hline
\end{tabular}

\footnotetext{
${ }^{14}$ En el bésiro, según Sans (2010), la palatalización ocurre regularmente solamente en el ambiente $i_{-} a$. La consonante / $/ \mathrm{k} /$ se palataliza también en el ambiente $i \_o$, mientras que /t/ se palataliza entre $i$ y cualquier vocal no anterior $(a, o, \bar{u}, \dot{i})$. La fricativa /s/ se palataliza tras $i$ sin importar la vocal que le sigue, compartiendo este comportamiento con la / $\mathbf{x} /$ del migueleño (que corresponde regularmente a la /\$ / del bésiro). En cuanto al chiquitano ignaciano, Ciucci y Macoñó Tomichá (2018) observan que la palatalización progresiva no ocurre si a la consonante le siguen / i i/; además, la palatalización de /p m/ en el ambiente $i \_o$ está sujeta a determinadas restricciones morfológicas.
} 


\begin{tabular}{|c|c|c|c|}
\hline$|\mathrm{ti} \boldsymbol{\beta} \mathbf{i} \mathbf{i} \mathbf{i} \mathbf{i}-\mathbf{x} \dot{\mathbf{t}}|$ & /tißָiß̦íci// & 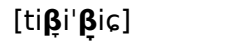 & 'tibibi (O)' \\
\hline |tosißָi-ko| & /tosíß̄iço/ & [to'sißָiço] & 'está gritando' \\
\hline |urasißָi-xí| & /urasiß̄íci/ & [urasi' $\boldsymbol{\beta}$ īi ] & 'sardina' \\
\hline |arißָironع| & /arißָiróne/ & [arißָi'rơ:nع] ] & 'almidón' \\
\hline |kißָitjo-xí| & /kißָitfóxít/ & [kißָi't]ox] & 'camote' \\
\hline $\mid \varnothing-(\beta)$ a-c̃tonimi-?o| & /maz̃tonímiło/ & [maz్ñto'nimij?o] & 'se lava las manos' \\
\hline$\left|i_{2}-m i k o r a-x+1\right|$ & /miçoráxit/ & [miço'rax] & 'Nicolás (ठ̋)' \\
\hline
\end{tabular}

Finalmente, es importante mencionar aquí un fenómeno que podría estar relacionado con el bloqueo discutido arriba y que consiste en lo siguiente: cuando a un tema que termina en /i/ se le agrega uno de los sufijos |-pí| NEG/ DUBIT, |-ti| DIR O |-xi| $\times$, la vocal del sufijo se transforma en una /i/, como se muestra en (31).

\begin{tabular}{|c|c|c|c|}
\hline $\mid \mathrm{t} \mathrm{j} \mathrm{i}-\varnothing$-ura-p-oßi-pi| & /tكurapó?ipi/ & [tكura'pơ?ịpi]] & 'no avisó' \\
\hline |t ti-ix-õ?õ-ka-oßi-pł̇| & /tfiįiøõ?õkóripi/ & [t & 'no oigo (ㅇ)' \\
\hline |t $\int \mathrm{i}-\mathrm{ix}-$ suputa-ka-api-pł| & 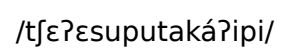 & 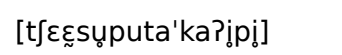 & 'no conozco' \\
\hline |tsai-pł| & /tsáipi/ & ['tsaipi]] & 'casi' \\
\hline $\mid \varnothing$-tsiro-ti?i-ti $\mid$ & /tsirotíriti/ & [tsiro'tiijtij] & 'se va $\left({ }^{\lambda}\right)^{\prime}$ \\
\hline $\mid i_{1}$-aruß̧api-xi $\mid$ & /aruß̦aríci/ & [aruß̦a'?ৃici (nana'?̧ux)] & 'es ancho (el chaco)' \\
\hline |ko r-oß-ori-xi| & /ko roßorí́i// & [koroßo'ríi $(r \varepsilon, ? ̧ \varepsilon)]$ & 'son hediondos (pues)' \\
\hline
\end{tabular}

En el caso de |-pi| NEG/DUBIT y |-tí| DIR es probable que el proceso de armonización esté motivado por la necesidad

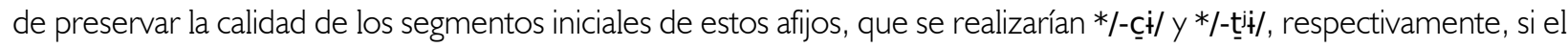
proceso de armonización no existiera. Se podría suponer que en la mente de los hablantes la preservación de la calidad de la consonante resulta más importante que la preservación de la calidad de la vocal. Sin embargo, esta explicación es algo problemática por no contemplar el sufijo |-xł| × y permanece especulativa.

\section{ESTATUS DE $\boldsymbol{s}$}

Hay evidencias que sugieren que el segmento [s] podría analizarse como un alófono del fonema /r/ en la posición de coda. Crucialmente, en algunos morfemas que claramente contienen $|\boldsymbol{r}|$ este segmento puede alternar con [s] en los contextos de elisión vocálica (ver 'Ensordecimiento y elisión'), como se muestra en (32) a continuación.

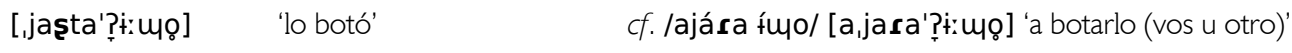

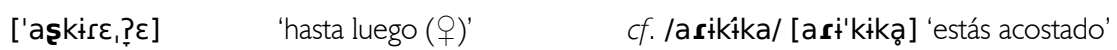

$$
\begin{aligned}
& \text { (lit. 'acostate pues') }
\end{aligned}
$$

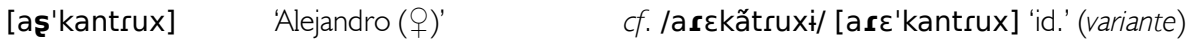

Otros ítems no presentan alternancias sincrónicas (o por lo menos no se han atestiguado las respectivas alternantes con $/[/$ ), pero en sus cognados en otras variedades del chiquitano —o en sus étimos en el caso de los préstamos-a la /s/ en coda del chiquitano migueleño le corresponde / $/$ / ( $y$ en el caso del castellano, cualquier consonante líquida o el alófono [ơ] del fonema /d/), como se muestra en (33). 
(33)

\begin{tabular}{|c|c|c|}
\hline \multirow{3}{*}{$\begin{array}{l}\text { [asko'res] } \\
\text { [joscaki'jox] }\end{array}$} & 'caracoré' & cf. lomeriano $n$-arako're-s 'id.' (Sans, 2 \\
\hline & 'paquió' & cf. ignaciano de San Rafael jo:rip'aki'?o- \\
\hline & & n-oripia'kjo-s 'id.' (Sans, 2010, p. 160) \\
\hline [şoß̦ $\left.\varepsilon \mathbf{s}^{\prime} t o ̛ x\right]$ & 'Roberto' & $<$ cast. Roberto \\
\hline ['ßָع:stę] & 'verde' & $<$ cast. verde \\
\hline [koski' ro:: rદ̧] & 'corregidor' & $<$ cast. corregidor ${ }^{15}$ \\
\hline [as'pơrkax] & 'alforja' & $<$ cast. alforja \\
\hline [as'katç] & 'alcalde (+)' & $<$ cast. alcalde \\
\hline [as'mentrax] & 'almendra' & $<$ cast. almendra \\
\hline ['meșkụ] & 'médico (q)' & < cast. médico \\
\hline
\end{tabular}

Obsérvese que los mismos fonemas líquidos del castellano $\left(/ r /, / \bar{r} /, / I /, / K /^{16}\right.$, bien como el alófono [ơ] del fonema /d/ o cualquier secuencia de éstos) se sustituyen por / / / en la posición de ataque en los préstamos (el alófono [d] del fonema /d/ del castellano se adapta consistentemente como /t/), como se muestra en (34) a continuación.

\begin{tabular}{|c|c|c|}
\hline [mate'rax] & 'bandera' & $<$ cast. bandera \\
\hline [sano'rax] & 'señora' & < cast. señora \\
\hline [arc'kantrux as'kantrux] & 'Alejandro (+)' & $<$ cast. Alejandro \\
\hline [iøkars'rax] & 'escalera' & $<$ cast. escalera \\
\hline [ro'rẽysax] & 'Lorenza' & < cast. Lorenza \\
\hline [to'rơric] & 'Dolores' & $<$ cast. Dolores \\
\hline [ma'iøti, ru] & 'profesor (q)' & $<$ cast. maestro \\
\hline [as'mentrax] & 'almendra' & $<$ cast. almendra \\
\hline [ka:su'xi6] & 'Carlos (P)' & $<$ cast. Carlos \\
\hline [karu'me: rụ] & 'Carmelo (P)' & $<$ cast. Carmelo \\
\hline [pe'sux] & 'Pedro (+)' & $<$ cast. Pedro \\
\hline ['rikox] & 'rico' & $<$ cast. rico \\
\hline [ra'simox] & 'racimo' & < cast. racimo \\
\hline [ßָuri'çax] & 'mula' & $<$ cast. burrica \\
\hline [a'rox $]$ & 'arroz' & $<$ cast. arroz \\
\hline [ira'pąㄷaㅁ] & 'Rafael (P)' & $<$ cast. Rafael \\
\hline ['rimax] & 'lima’ & $<$ cast. lima \\
\hline 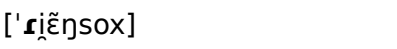 & 'lienzo’ & $<$ cast. lienzo \\
\hline [sa'rinax] & 'Salinas' & $<$ cast. Salinas \\
\hline [ãnkع'rax] & ‘Ángela’ & < cast. Ángela \\
\hline [kata'sinax] & 'Catalina' & < cast. Catalina \\
\hline
\end{tabular}

\footnotetext{
${ }^{15}$ A pesar de que en el castellano camba moderno este ítem contenga una fricativa ([kosẹehi'ōor $\left.\mathrm{C}\right]$ ), la adaptación de los demás segmentos en este préstamo es típica de un estrato más antiguo de préstamos, en el que el fonema $/ \bar{r} /$ del castellano se sustituye normalmente por la $/ \mathbf{r} /$ del chiquitano (probablemente esos préstamos se incorporaron al chiquitano en una época cuando el fonema / $/ \bar{r} /$ todavía conservaba una realización vibrante): /aróxit < cast. arroz, /ríkoxit < cast. rico, /rasímoxi/ < cast. racimo. En los préstamos más

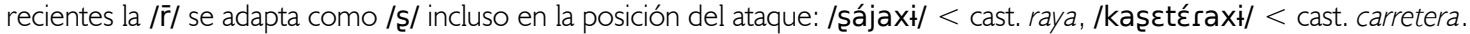

${ }^{16}$ En algunos préstamos la / $/ /$ del castellano se adapta como /j/, como, por ejemplo, en /kájaxi/ < cast. calle.
}

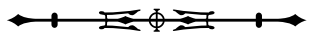




\begin{tabular}{|c|c|c|}
\hline [uru' $\left.\beta^{w i}: 6\right]$ & 'Luis (P)' & $<$ cast. Luis \\
\hline [pi'ripị] & ‘Felipe' & $<$ cast. Felipe \\
\hline 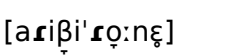 & 'almidón' & < cast. almidón \\
\hline [samĩ̄j]:: rદ̧] & 'San Miguel' & < cast. San Miguel \\
\hline [miço'rax] & 'Nicolás' & $<$ cast. Nicolás \\
\hline [saßz'rax] & 'Isabel' & $<$ cast. Isabel \\
\hline [kaxtri'janux] & 'castellano' & $<$ cast. castellano \\
\hline [ $\tilde{\varepsilon} \eta S \varepsilon n t \varepsilon ' \boldsymbol{r o ̛ : ~} \mathbf{s}$ ] & 'yesquero' & $<$ cast. encendedor \\
\hline [koski'rơ: $\boldsymbol{s \varepsilon g ] ~}$ & 'corregidor' & $<$ cast. corregidor \\
\hline [kanti'rax] & 'Cándida' & < cast. Cándida \\
\hline
\end{tabular}

Se podría hipotetizar que los segmentos líquidos y [ơ]] del castellano se adaptan siempre como /r/ (34), que se realiza como [s] en la posición de coda (33). Sin embargo, hay datos que muestran que, sincrónicamente, no existe una distribución perfectamente complementaria entre la / / / y la /s/ en el chiquitano migueleño, ya que se han atestiguado ocurrencias de [r] en la posición de coda — tanto en los préstamos adaptados (35a-b) como en los contextos de elisión vocálica (35c) — y de [s] en ataques en algunos préstamos (36), como se mencionó en la nota 16.

\begin{tabular}{|c|c|c|c|}
\hline a. & [osßar'tox] & 'Osvaldo (o)' & $<$ cast. Osvaldo \\
\hline b. & [mer'se: ra] & 'Mercedes' & $<$ cast. Mercedes \\
\hline - & ['tarko 'taruko] & 'muy' & \\
\hline
\end{tabular}

\begin{tabular}{|c|c|c|c|}
\hline a. & [kaş $\left.\varepsilon^{\prime} t \varepsilon r a x\right]$ & 'carretera' & $<$ cast. carretera \\
\hline b. & ['sajax] & 'raya’' & $<$ cast. raya \\
\hline c. & ['xaşax] & 'caneco' & $<$ cast. jarra \\
\hline
\end{tabular}

Por lo menos algunos de los préstamos supracitados son de uso totalmente consolidado en el chiquitano migueleño (35a-b, 36c); obsérvese que las formas de los nombres propios en (35a-b) difieren significativamente de las de sus étimos en castellano. En este trabajo, se optó por considerar /s/ un fonema marginal del chiquitano migueleño recientemente fonologizado, o tal vez en trance de fonologización $n^{17}$.

\section{ESTATUS DE $\boldsymbol{h}$}

No está absolutamente claro si realmente existe un contraste entre $/ \mathrm{h} /$ y $/ \mathrm{x} /$. En los materiales destinados a la comunidad chiquitana se ha representado ambos supuestos fonemas con un único grafema 〈j〉 (Nikulin, 2019a). Una dificultad adicional proviene del hecho de que $/ \mathrm{h} /$ aparece solamente en el habla fluida en determinados contextos morfosintácticos, resultando en la ausencia absoluta de ejemplos con /h/ en el corpus de palabras aisladas elicitadas. Algunos ejemplos que ilustran la ocurrencia de /h/ están en (37).

17 Un(a) evaluador(a) anónimo/a sugiere que las ocurrencias de [ $r$ ] en coda, como en (35), podrían considerarse excepciones léxicas de una regla que convierte / $\mathrm{r} / \mathrm{a}$ [s] en coda. Efectivamente, algunas teorías fonológicas ( $c$. Zonneveld, 1978) permiten especificaciones diacríticas de este tipo. Sin embargo, en este estudio no se aceptará esta posibilidad alternativa de análisis por no explicar las ocurrencias de [s] en ataques (36), además de tener la desventaja de exigir complicaciones innecesarias en la fonología. 
a.

\begin{tabular}{|c|c|c|}
\hline ['kotaa & noxo:'ne & haع्र'mo] \\
\hline /kótaPa & noxooné & haemó/ \\
\hline |kotaPa & r-oxoo-ne & h-a-cmo| \\
\hline dónde & L-doler-ATTR & $\mathbf{H}-2 \mathrm{sG}-\mathrm{DAT}$ \\
\hline
\end{tabular}

b.

[i6ĩ̄jaka
/iøináka
|ix-xina-ka
1sG.9-querer-F.SAP

'nơuwa?

nóuß̧a?a

r-ouß-a(?)a

L-3PL-comer hii' rọti

hiiróti

h-ix-iro-ti

H-1sG. 9 -ir.NF-DIR

kunumasima'?̣aka

kunumasimarákaa

kuruß̧asi-ma?a-kaa

pollo-DIM-PL
' 'apat $\int \varepsilon$

Gápat $\int \varepsilon$

ix-(ß)a-pat $\int \varepsilon$

1sG. P-ACT-buscar rose'jox

rosejóxł

r-osejo- $x+$

L-maíz-X

'Quiero ir a buscar maíz para que los pollitos coman mañana.'

c.

e.

\begin{tabular}{|c|c|c|c|c|c|}
\hline [tutsĩ: 'ja & 'him] & d. & [6ĩ̄]a'ne & ho'ne & țo'wițå \\
\hline /tutsiiná & hîmo/ & & /६înanć & honé & tojoß̦ítia/ \\
\hline |tutsi-Vna & $\mathrm{h}-\mathrm{i}-(\varepsilon) \mathrm{mo}$ & & |ixina-ne & hone & itoßָi-t \\
\hline saberse-STAT & $\mathrm{H}-3-\mathrm{DAT}$ & & frío-ATTR & antes & noche-? \\
\hline \multicolumn{3}{|l|}{ 'Él sabe.' } & \multicolumn{3}{|c|}{ '¿Hacía frío anoche?' } \\
\hline \multicolumn{2}{|c|}{ [t]itutsĩ: 'japirદ๕్ } & 'ho्रहm] & f. & {$[$ ta } & 'hapع̨] \\
\hline \multicolumn{2}{|c|}{ 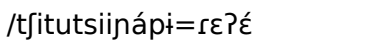 } & hoźmo/ & & $/$ ta & hápe/ \\
\hline \multicolumn{2}{|c|}{$\mid \mathrm{t} \int \mathrm{i}-\mathrm{tutsi}-\mathrm{Vna} a-\mathrm{p} \dot{\mathrm{a}}=r \varepsilon ? \varepsilon$} & h-o-(ع)mol & & ta & hapel \\
\hline \multicolumn{2}{|c|}{ NEG-saberse-STAT-NEG = pues..+} & $\mathbf{H}$-1INCL-DAT & & FOC & arriba \\
\hline \multicolumn{3}{|c|}{ 'No sabemos (incl.) pues.' } & & \multicolumn{2}{|c|}{ 'Sí [está] arriba.' } \\
\hline
\end{tabular}

'țowaką]

țóß̄aka/

itoß̧aka|

mañana. ${ }^{2}$

La /h/ móvil ocurre al inicio de algunas formas flexionadas de la adposición dativa (37a, c, e) y en las formas no finitas de los verbos (37b), siempre que éstos tienen por su segmento inicial una vocal. Además, es parte de algunos adverbios, como /(h)ape/ 'arriba' y /(h)one/ 'en el pasado' (37d, f) 18 . En esta etapa de la investigación no se puede proponer una generalización descriptiva que explique todas las (no) ocurrencias del supuesto fonema $/ \mathrm{h} /$ en el habla fluida. Su ocurrencia o no ocurrencia parece estar condicionada por el contexto izquierdo: /h/ suele ocurrir con bastante frecuencia tras los predicados atributivos en /-ne/ (37a, d), estativos en /-Vna/ (37c) y la partícula multifuncional /ta/ FOC/COP/sUB (37f), pero también en otros contextos (37b, e).

\footnotetext{
18 Un(a) evaluador(a) anónimo/a sugiere analizar las ocurrencias de /h/ como insertadas mediante una regla de epéntesis en palabras que inician con vocales. Sin embargo, es importante tener en cuenta que la mayoría de las palabras que inician con vocales (por ejemplo,

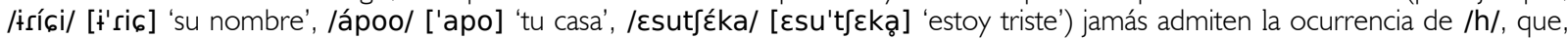
como ya se dijo, se limita a la adposición dativa, a las formas no finitas de los verbos y a algunos adverbios. Por ello, prefiero mantener el análisis en que las palabras que admiten la ocurrencia de /h/ presentan la respectiva especificación.
} 
Una posibilidad que merece ser investigada en el futuro es que la consonante [h] emerge en la superficie a través de un proceso de sandhi, cuando un morfema que termina en una |h| subyacente (por ejemplo, |-nch| ATTR, |-Vnah| STAT, |tah| FOC/COP/SUB) es seguido por un morfema que empieza con una |h| subyacente (por ejemplo, |hape| 'arriba'). Sin embargo, la ocurrencia de [h] en ejemplos como (37b, e) permanecería sin explicación: son los únicos ejemplos

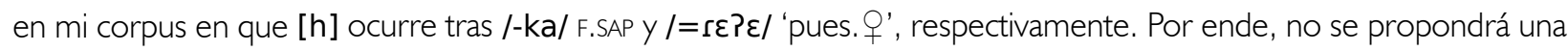
explicación universal para todas las instancias de /h/ hasta que un corpus más extenso sea analizado.

\section{RESOLUCIÓN DE HIATOS Y ESTATUS DE $\boldsymbol{\eta}, \boldsymbol{u}$}

Una inspección del léxico registrado muestra que el chiquitano migueleño no presenta secuencias del tipo $V_{\text {[+alta] }} \bigvee_{\text {[-anterior] }}$ (ni tampoco las secuencias $* i u, * u \dot{*}, * u i$ ) en la superficie en el interior de palabras. Por el otro lado, se destaca la alta

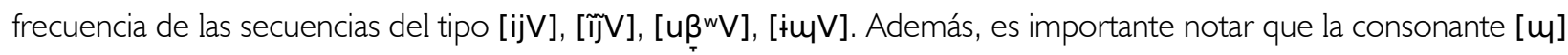

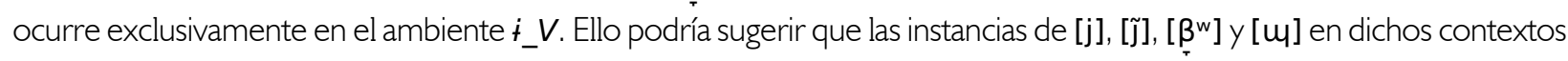
deban analizarse como automáticas, es decir, que una regla fonológica de epéntesis inserte una aproximante tras vocales altas seguidas de otra vocal que no sea alta ni anterior, así como en los ambientes $i_{-} u, u_{-}+$il (38). Obsérvese que ello permitiría reducir el inventario de segmentos consonánticos a través de la eliminación de / $\mathbf{m / :}$ todas las ocurrencias de ese segmento podrían atribuirse a la regla de epéntesis (por ejemplo, /ióxi// [i'uọx] 'urina. ơ').

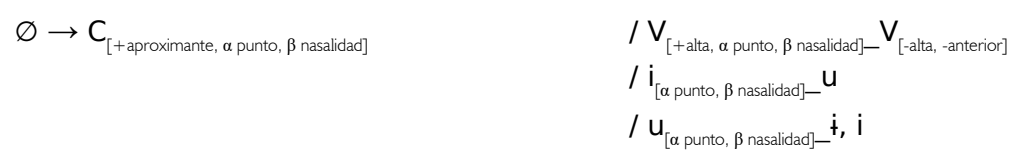

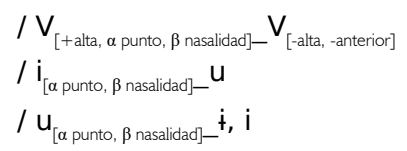

\begin{tabular}{|c|c|c|c|}
\hline a. & /Biotsíxí/ & [ßijo'tsix] & 'bibosi (o)' \\
\hline b. & /kĩána/ & [kĩ'ja:na] & 'debajo de' \\
\hline c. & /kuruasíxí/ & 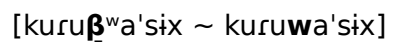 & 'pollo (P)' \\
\hline d. & /kiónotsoßo/ & [ki'uơnotso?] & 'es tuerto' \\
\hline e. & /ț̣uíci/ & 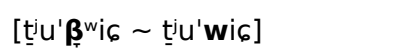 & 'concha' \\
\hline
\end{tabular}

Sin embargo, a pesar de que se haya tenido en cuenta la regla propuesta en (38), el autor prefiere abstenerse de utilizarla, y más bien derivar las formas en (38a-d) de una representación fonológica menos abstracta, como se muestra en (38'), por motivos que se explicarán a continuación.

\begin{tabular}{|c|c|c|c|}
\hline a. & /ßָijotsíxí/ & [ß̧ijo'tsix $x]$ & 'bibosi (P) \\
\hline b. & /kinána/ & [kî'j̃a:na] & 'debajo de \\
\hline C. & /kuruß̦aśxxt'/ & [kuruß̧wa'six $\sim$ kuruwa'six] & 'pollo (\%)' \\
\hline & /kimónotso?o/ & [ki'mơnotso?] & 'es tuerto' \\
\hline & /țußßí́িi/ & 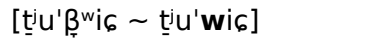 & 'concha' \\
\hline
\end{tabular}

El primer argumento consiste en el hecho de que las secuencias [ijV], [iٓjV] y [uß $\beta^{w} \mathrm{~V}$ ] ocurren también en palabras morfológicamente complejas en que se puede demostrar que las aproximantes son realizaciones de fonemas consonánticos (39). Lo contrario no ocurre: no se sabe de ninguna palabra cuya composición morfológica nos obligue a analizar como epentéticas las aproximantes que son parte de secuencias de ese tipo. 
(39)
a. $\quad \mid i \frac{x}{j}-\beta$ aitsi|
/ijáitsi/
[i'jaitsi]
'mi hamaca'
b. $\quad \mid i \frac{x}{j}$-masu|
/inásu/
[ĩ̃jasụ]
'mi oreja'
c. |o-ßָaki-xi|
/uß̦akí́i/
[uß̧wa'kig]
'garza $\left({ }^{\lambda}\right) '$

El segundo argumento proviene de las particularidades de la operación del proceso morfofonológico de nasalización. Este proceso de asimilación a distancia, desencadenado por una clase cerrada de sufijos (notablemente, /-maPa/ DIM), afecta las consonantes / $\beta$ г j u/, transformándolas en /m n n $\mathrm{\eta} /$. Las secuencias /ijV/, /uß̦V/, /iulV/ también se ven afectadas (40).
a. $\quad \mid$ kijara-mara|
/kinanámara/
[kĩ̄a'nama?]
'zorrito (ㅇ)'
b. |kurußasi-mara|
/kunumaśmara/
[kunuma'sima?]
'pollito (ㅇ)'
c. |kimo-mara|
/kinómara/
[ki'yọma?]
'urucucito (+ + )'

El proceso de la nasalización debe clasificarse como léxico y no posléxico, ya que hay casos en que consonantes nasales coocurren con / $\beta$ г j u/ en una misma palabra: /kimónotso?o/ [ki'mọnọtso?] 'es tuerto', /rominúxí/ [romi'nux]

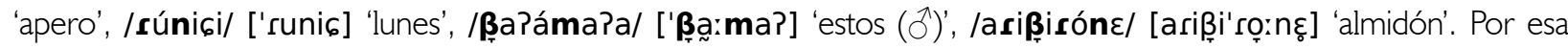
razón, el segmento $[\boldsymbol{u}]$ se atribuye al fonema / $\boldsymbol{u} /$ en vez de que se le dé una interpretación epentética: si el proceso de epéntesis fuera posléxico, su resultado no podría ser afectado por un proceso léxico.

Es oportuno mencionar un fenómeno similar al proceso de epéntesis propuesto (y rechazado) arriba, este sí automático. Consiste en un aumento del grado de constricción hacia la fase final de /i u/ precediendo una / / /, resultando — desde el punto de vista segmental — en la ocurrencia de una aproximante ultracorta inmediatamente antes del cierre de la glotis, que se transcribirá como [j/w], como en /páki?o/ ['pakii?o] 'se cayó', /pi?úxxi/ [pij'?̣ũx]

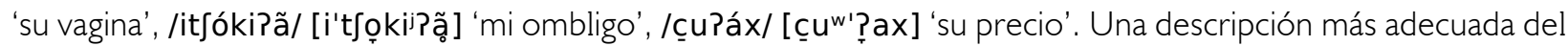
fenómeno podría hacerse en los términos de la coordinación gestual (cf. Gafos, 2002); aparentemente la fase final del gesto del cuerpo de la lengua que corresponde a /i u/ no está sincronizada con ninguna fase de / $/$ /, sino con la fase inicial de la vocal siguiente. De esta forma, la transición de /i u/ hacia la vocal siguiente estaría sobrepuesta al gesto de la oclusión glotal y por ende se volvería parcialmente inaudible. El autor tiene la intención de hacer mediciones exactas con la finalidad de determinar el grado de la superposición gestual en las secuencias del tipo /iPV, uPV/ en futuros trabajos.

\section{VOCALES}

En cuanto a las vocales del chiquitano migueleño, se postula aquí un inventario simétrico de 12 fonemas vocálicos (6 orales y 6 nasales), que se encuentran expuestos en la Tabla 2.

Tabla 2. Las vocales del chiquitano migueleño.

\begin{tabular}{c|c|c|c}
\hline & Anteriores, no redondeadas & No anteriores, no redondeadas & No anteriores, redondeadas \\
\hline Altas & $\mathrm{i} \tilde{\mathrm{i}}$ & $\dot{\dagger} \tilde{\mathrm{t}}$ & $\mathrm{u}$ ũ \\
\hline No altas & $\varepsilon \tilde{\varepsilon}$ & $\mathrm{a}$ ã & o õ \\
\hline
\end{tabular}


En (41-47), se muestran algunos contrastes fonológicos con pares mínimos o análogos entre las vocales orales. Por razones de espacio, se incluyen solamente los pares de segmentos que difieren en una única dimensión del cuadro.

$/ 0 / x / 4 /:$

$\mid \mathrm{a} / \mathrm{x} / \mathrm{t} / \mathrm{:}$

$/ \varepsilon / \times / i /:$

$10 / \times / a /:$

$/ \mathrm{u} / \mathrm{x} / \mathrm{i} / \mathrm{s}$

$\mid a / x / \varepsilon /:$

/ $/ \mathrm{x} / \mathrm{i} /$

$$
\begin{aligned}
& \text { [ß̧ijo'tsix] 'bibosi (우)' } \\
& \text { [o'tox] 'su sangre' } \\
& \text { [a'rox] 'arroz' } \\
& \text { [țo'o'pinwig] 'noche' } \\
& \text { [to'rox] 'toro' }
\end{aligned}
$$

[ka'?̦ax] 'piedra'

[a'ric] 'aji'

[o' $\beta^{w} \mathbf{a k i i ? o ]}$ 'está abollado'

[na'matą] 'lo abrió

[matc' rax] 'bandera'

[ß̧عja' Box] 'bagre (P)'

[arcjo'kox] 'su llanto'

[u' $\beta^{\mathrm{w}} \varepsilon$ ? $]$ ] 'afuera'

[mع'sax] 'mesa'

[ยna'kic] 'garabatá (†)'

[o'nes] 'jone'

[o'ric] 'su olor'

[paú'sox] 'puquio, paúro'

[sij'?ơ] 'cotorra (P)'

['oem] 'a nosotros (incl.)'

[apa'?̧ux] 'su sobaco'

[ju'pux] 'su padre (de mujer)'

[ça'ux] 'su mano izquierda'

[ja'ux] 'su animal'

[tsa'uka] 'bárbaros, ayoreos ()'

[pa'tax] 'alacrán (P)'

[a'sux] 'su hoja'

[i'tsax] 'su rodilla'

[pa'?̦ic] 'tabaco'

[pa'?̣ana?] 'humedito'

['íri] 'tu nombre'

[țiaki] 'la chala de'

['i'Pij] 'tu orina'

['ai] 'tu hijo'

['içaí] 'mi leche'
[ iiju'tsix] 'isotoúbo (ㅇ)', 'murciélago (ㅇ)' (San Juan) [o'tux] 'su lengua'

[a'rux] 'su labio'

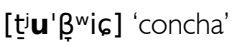

[tu' rux] 'turu'

[kít? ix ] 'tierra'

[+'ric] 'su nombre'

[o' $\beta^{w} \mathbf{i}$ kir?o] 'se cuece, está cocido'

[ni'mơtą 'le contestó'

[mato' $r \mathbf{i x}$ ] 'tarechi ( $(+)$

['ßijaßßo] 'se ríe'

[xorijo'kox] 'soriocó (P)'

[purusu' $\beta^{w i}$ i] 'blanco'

[kami'sax] 'camisa'

[iٓja'kax] 'corechi (o)'

[a'nes] 'carne'

[a'ric] 'aji'

[tau' rax] 'tabla'

[si'jax] 'silla'

[a'عm] 'a vos'

[pa'?̦ix] 'mujer'

[kí'pix] 'mosca (P)'

[ça'ix] 'su leche'

[ja'ix] 'su padre (de hombre)'

[tsa'íka] 'mis hijos' (en sermones)

[pe'tax] 'peta, tortuga ( $($ )'

[ $\boldsymbol{\varepsilon}$ 'sux] 'su cara, su rostro'

[i'tf $\varepsilon$ ] 'lejos'

[pe'?ị] 'costilla'

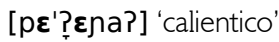

['iri] 'nada, no'

[țiaki] 'la piel de, la cáscara de'

['i:] 'mi canilla'

['arị] 'tu boca'

['içarị] 'mi hueso' 
En cuanto a las vocales nasales, son relativamente raras en el chiquitano migueleño, volviendo difícil la tarea de encontrar pares mínimos o análogos que las involucren. En (48) se ilustra con un número reducido de ejemplos el contraste entre vocales orales y nasales.

$$
\begin{aligned}
& \text { [pa'?ৃi } \mathbf{i} \text { ] 'tabaco' } \\
& \text { [a'?̧ic] 'su boca' } \\
& \text { ['paíro] 'mama' } \\
& \text { [ta'?̧ux] 'barro, lodo' } \\
& \text { [motojo'?̣ عs] 'motoyoé (ㅇ)' } \\
& \text { ['ica?] 'a comer yo' } \\
& \text { [ع'tso?o? 'mi diente' } \\
& \text { [pơ'?ִָox] 'gallo, macho de pato' }
\end{aligned}
$$

$$
\begin{aligned}
& \text { [pa'?ָ̃̃ ] 'cura, padre' } \\
& \text { [xa'?ָĩ ] 'puchi' } \\
& \text { ['pa:̊x] 'mes' } \\
& \text { [pij'?ִũx] 'su vagina' } \\
& \text { [60'?ָ̃̃s] 'campo, pampa' } \\
& \text { [i'६ã?å̃] 'mi puchi' } \\
& \text { [ع'tsỡ? ỡ] 'a morir yo' } \\
& \text { [takõ̃'? ?ָõx] 'tatú (o)' }
\end{aligned}
$$

En (49) se ilustran algunos contrastes entre las propias vocales nasales.
['ã?ą̃] 'tu puchi'

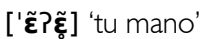
['ō̃õo] 'a vomitar vos'
['ỡyką] 'vomitaste'
['ũyka] 'quién (+)'

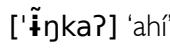

['ĩnçatị] 'me voy'

\section{REALIZACIÓN FONÉTICA}

Las vocales del chiquitano migueleño presentan relativamente poca alofonía. Los símbolos del AFI [i i i † $u$ u ũ $\varepsilon$ $\tilde{\varepsilon}$ o õ a ã] representan la pronunciación prototípica de las respectivas calidades vocálicas de forma bastante exacta (sin tomar en cuenta el fenómeno de ensordecimiento, que se discutirá en la subsección siguiente, y los alófonos no silábicos, que se presentarán en la subsección 'Silabificación'), con las siguientes salvedades:

(i) las vocales /o õ/ suelen pronunciarse como medias [ơ ỡ] en las sílabas tónicas y como medias altas [o õ] en las sílabas átonas ([a'rơx] 'arroz', [o'nes] 'jone'), aunque la diferencia entre los alófonos es mínima (no se la ha cuantificado instrumentalmente);

(ii) en el subdialecto de San Juan, pero no en el de San Miguel, la vocal /ł/ suele realizarse como redondeada $[\forall]$ si la sílaba siguiente contiene una / $\mathrm{u} /$ (o asimilarse totalmente si no interviene ninguna consonante), como

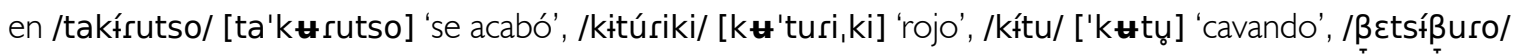

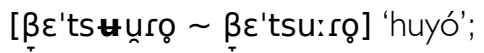

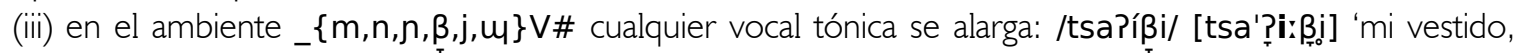

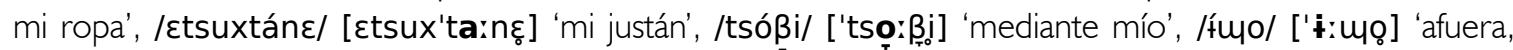
abriendo', líno/ ['iznọ] 'para mí ()'.

Además, la nasalidad en las vocales nasales se puede manifestar en la superficie de diversas maneras, dependiendo del ambiente. La realización que juzgo ser más básica, [Ṽ], ocurre en tres ambientes: precediendo una / $/$ /, precediendo una / $/$ / o siguiendo una / $/$ / (50). En esos casos, la nasalidad suele ser bastante débil (no se ha podido medirla con nasómetro).

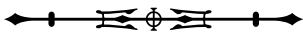




\begin{tabular}{|c|c|c|c|}
\hline \multirow[t]{6}{*}{ a. } & /țjokî?okóta/ & [țokĩiPo'kota] ] & 'lo está desnudando' \\
\hline & /tsom干̃r̃̃ / & 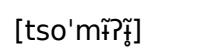 & 'nosotros (excl.)' \\
\hline & 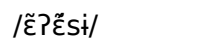 & 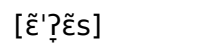 & 'su mano' \\
\hline & /çakãrãx/ & [çakã'?̣ãx] & 'su hígado' \\
\hline & /õPốpakł/ & [õ'?ָõpakị] & 'tu hombro' \\
\hline & /6õ?õkóPi/ & [6õ?ָõ'kợị] & 'yo escucho (P)' \\
\hline b. & /tamõxíci/ & [tamõ'xi६] & 'chuubi, falcón (P)' \\
\hline \multirow[t]{10}{*}{ c. } & /arīca/ & [a'?ָīตą] & 'tu tocayo' \\
\hline & /nonicarīci/ & [nonica'?ָī́] & 'sucupiro' \\
\hline & /parī́i/ & [pa'?ָī́] & 'cura' \\
\hline & /xarī́i/ & [xa'?ָī6] & 'puchi' \\
\hline & /nař̃mara/ & [na'?ָ̃̃ma?] & 'niño' \\
\hline & /astanaPũka/ & [astana'?̣ũką] & 'vos ves bien' \\
\hline & /pi?úxit/ & [pij'?ָũx] & 'su vagina' \\
\hline & /60?Ẽsi// & [60'?ָع̃s] & 'campo, pampa' \\
\hline & /õ?õsikíxt/ & [õ?̃õși''kix] & 'su aro' \\
\hline & /itfóki?ã/ & [i't5ookii\}ạ̃] & 'mi ombligo' \\
\hline
\end{tabular}

En todos los demás ambientes las vocales nasales adquieren en su fase final una constricción en la cavidad oral (en términos segmentales, una consonante nasal de transición). El punto de la constricción es homorgánico con la consonante siguiente si ésta es una oclusiva o una africada (51a). Si la consonante siguiente es una nasal o una fricativa (o si no hay una consonante), la consonante nasal de transición será velar (51b) 19. Es importante notar que la nasalidad de las vocales se vuelve extremadamente débil delante de las consonantes de transición [ $\mathrm{m} n$ ] (no se representará en las transcripciones fonéticas), pero muy audible delante de [n, $\mathbf{\eta}]$. Ello indica que el gesto del rebajamiento del velo del paladar no se sincroniza solamente con la fase inicial de la vocal, sino que puede sincronizarse con el gesto de cierre de la articulación de la consonante siguiente.

a.

\begin{tabular}{|c|c|c|}
\hline /kãpoxì/ & ['kampox] & 'espacio' \\
\hline /ţốpaxit/ & ['trơmpax] & 'chompa' \\
\hline /tãtóxit/ & [tan'tox] & 'güembé' \\
\hline /ह̃tußo/ & ['Entußo] & 'cuántos' \\
\hline /aß̦arižt $\varepsilon /$ & 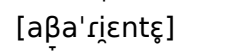 & 'aguardiente' \\
\hline /çoốto?o/ & ['çơ:nto?] & 'luego' \\
\hline /sútsaßָo/ & ['sun,tsa & 'es hediondo' \\
\hline /ßָz̃taxt/ & ['ßֲntax] & 'venta, tienda' \\
\hline /matãtfómeno/ & [matanַ'ttomeñ] & 'está ordeñando' \\
\hline /íçatt/ & ['ĩnַçatị] & 'me voy' \\
\hline /tomĩço/ & [to'mĩnַço] & 'domingo' \\
\hline
\end{tabular}

\footnotetext{
19 Las vocales nasales no ocurren precediendo /n/. En este ambiente, las vocales nasales subyacentes parecen desnasalizarse regularmente:
} |kõõ-jol /kóono/ 'se muere', |pokunũ-Vna| /pokunuuná/ 'contento'. 


\begin{tabular}{|c|c|c|c|}
\hline & /ốka/ & ['ō̃yką] & 'vomitaste' \\
\hline & /apokunúka/ & [apoku'nũnką] & 'estás contento' \\
\hline & /عtsốka/ & [ع'tsỡnkä] & 'me muero' \\
\hline & /mõkóxt/ & [mõy'kơx] & 'Mojos' \\
\hline & /tãkusíci/ & [tãnku'sic] & 'seboí (+)' \\
\hline & /ú́ka na?a/ & ['ũnkana,ã ] & 'quién es (\%)' \\
\hline & /łkaPa/ & ['ז̃nka?] & 'ahí' \\
\hline b. & /pã/ & ['pãn] & 'pan' \\
\hline & /sãkuã/ & [sãn'kuãa] & 'San Juan' \\
\hline & /tãma?/ & ['tãnma?] & 'pájaro' \\
\hline & /kãmará/ & [kãnma'?̣a] & 'ahora' \\
\hline & /ốno/ & ['ỡyno] & 'vomitó' \\
\hline & lakãsáka/ & [akãn'saką] & 'estás descansando; buenas tardes' \\
\hline & /riẽsoxit/ & ['riñ̃nsox] & 'lienzo' \\
\hline & /mapẽsara/ & [ma'pẽysarą] & 'piensa' \\
\hline
\end{tabular}

En cuanto a las secuencias vocálicas tautosilábicas (ver 'Silabificación') cuyo último elemento es una vocal nasal,

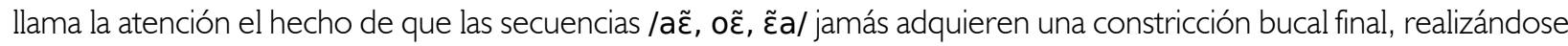

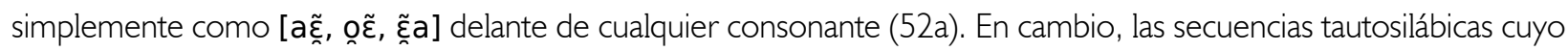
segundo elemento es una vocal nasal alta (/aĩ ã̃ aũ oĩ/) adquieren una constricción bucal final delante de las consonantes (incluyendo / $\mathrm{x} /$ pero excepto / $/$ /, 52b).

\begin{tabular}{|c|c|c|c|}
\hline \multirow[t]{3}{*}{ a. } & /6akiçaz̃ka/ & [6aki'çaz̃nką] & 'estoy cosiendo (P) \\
\hline & /mac̃tonímißo/ & [maz్̃to'nimii?o̊] & 'se lavó las manos' \\
\hline & /mac̃tapáxi?o/ & [mažnta'paxii?o] & 'lastimó la mano' \\
\hline \multirow{8}{*}{ b. } & /nac̃táma?a/ & [na登'tama?] & 'solito $(\widehat{\jmath}) '$ \\
\hline & /ponoz̃tóxi// & [ponoz̃'toox] & 'cinturón' \\
\hline & /ع̃ána/ & ['䈍a:na] & 'entre (líquido)' \\
\hline & /máĩkißo/ & ['maĩnkii?o] & 'se lavó las manos' \\
\hline & /aĩçá?i/ & [aĩnn'ça?i] & 'itraelo!' \\
\hline & /napã̃káxt/ & [napa苂'kax] & 'su mentira' \\
\hline & /maũxókono/ & [maũ̃n'xơkono] & 'está enfermo' \\
\hline & /țítmoĩțiaPáxít/ & [țimoĩnnița'?̦ax] & 'mitad' \\
\hline
\end{tabular}

Teniendo en cuenta que lo que analizo como vocales nasales se realiza como secuencias del tipo "vocal + consonante nasal" con gran frecuencia, uno podría preguntarse si éstas no se deberían analizar como secuencias de una vocal (oral) y una consonante nasal en coda (no especificada para el punto de articulación), permitiendo eliminar las vocales nasales del inventario. Sin embargo, tal análisis no se justifica para el chiquitano migueleño por las siguientes razones:

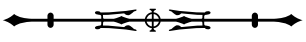


(i) sería imposible atribuir la nasalidad vocálica a una supuesta coda subyacente en ejemplos como /ع̃ána/ [' 'ূ̃a:na] 'entre (líquido)', en que una vocal nasal es seguida por otra vocal;

(ii) a diferencia de lo que ocurre en lenguas como el portugués o el francés, en chiquitano las vocales nasales jamás alternan con secuencias de vocales orales y consonantes nasales en el ataque de la sílaba siguiente;

(iii) el chiquitano migueleño presenta alternancias morfofonológicas entre vocales orales y nasales desencadenadas por segmentos nasales a distancia (por ejemplo, los prefijos $\mid$ tsoij-| 1EXCL e $\left|\mathrm{i}_{2}-\right|$ HT poseen los alomorfos /tsoĩ-/ e /-ĩ-/ que ocurren cuando la raíz contiene una consonante nasal: /tsoĩtfimóka/ 'estamos sentados (EXCL.)', /aĩçáPi/ 'itraelo!'); sería más natural atribuirlo a un proceso de propagación del rasgo [+ nasal] que a una supuesta inserción de una coda nasal subyacente;

(iv) en algunas pocas palabras, las consonantes nasales pueden ocupar la posición de coda como resultado de una elisión (morfofonológica) de una vocal: /masuunkóono/ [masu:n'kọ:ṇ] 'está barriendo', /masuunkotóxit [masu:nko'tox] 'escoba'; si se supusiera que las nasales en coda se elidieran al nasalizar el núcleo, habría que representar dichos lexemas como /masuunVkóono/, /masuunVkotóxit, pero la supuesta vocal jamás se pronuncia, ni siquiera en el habla lenta (aunque probablemente sí es parte de la representación morfofonológica de la raíz 'barrer').

Por ende, se concluye que el chiquitano migueleño posee doce vocales y no seis.

\section{ENSORDECIMIENTO Y ELISIÓN}

Todas las vocales átonas en el chiquitano migueleño se pueden ensordecer — y normalmente se ensordecen — ante pausa y en algunos casos en la posición final de una palabra fonológica en el interior de las frases (posiblemente delimitando los márgenes de las palabras prosódicas; no se han investigado detalladamente las condiciones del ensordecimiento en el interior de las frases $)^{20}$. Doy algunos ejemplos en (53a). Si la palabra fonológica termina en una secuencia de vocales átonas, el ensordecimiento jamás ocurre; la secuencia de vocales idénticas típicamente se realiza como una vocal corta sonora (53b).

\begin{tabular}{|c|c|c|c|}
\hline \multirow[t]{7}{*}{ a. } & /iøáka/ & [i'caką] & 'comí' \\
\hline & |हี? $\tilde{\varepsilon} /$ & 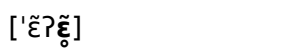 & 'tu mano' \\
\hline & /amásu/ & [a'masü] & 'tu oreja' \\
\hline & /marmatakúrutso/ & [maદ̧mata'kurutso] & 'está esperando' \\
\hline & /it\}óki?ã/ & [i'tكơkij?å̃] & 'mi ombligo' \\
\hline & /Kíjłßָo/ & ['kijiß̦o] & 'nació’' \\
\hline & /ariß̦iróne/ & [arißָi' rọ:nع̨] & 'almidón' \\
\hline \multirow[t]{3}{*}{ b. } & /ítfï/ & ['itff] $\left(*\left[' i t f f_{j}\right]\right)$ & 'mi cuello' \\
\hline & /ápoo/ & ['apo] (*['apo]) & 'tu casa' \\
\hline & /útsaí/ & ['utsai] (*['utsaị]) & 'nuestro.INCL hijo' \\
\hline
\end{tabular}

\footnotetext{
20 En algunos estilos del habla (supongo que sobre todo cuando el hablante desea comunicar la idea del cansancio), el ensordecimiento de una vocal alta puede ser acompañado por una considerable constricción oral. En este caso, las vocales /i i u/ se realizan como [ç x M],

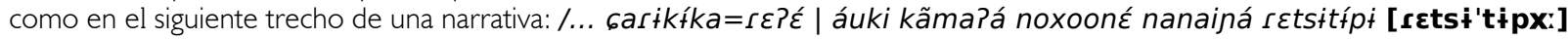
| oxoonź na?=itfárant ... / ' ... me acosté y de ahí en ese momento dolió todo mi cuerpo, dolió mi cabeza ....'.
} 
En algunos casos se notó un ensordecimiento total o parcial de la consonante que precede a la vocal ensordecida. Los casos más claros de este fenómeno en el corpus grabado se refieren a la /r/ (por ejemplo, /ß̧arákíro/ [ba'rikir̊o] 'está acostado, echado'), pero en el habla espontánea se han observado algunas ocurrencias del mismo fenómeno en

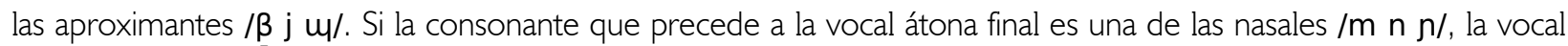
típicamente no se ensordece, sino que se elide completamente, sobre todo si es una de /a o ł/ (pero no /ع/; para /i u/ no hay ejemplos ni contraejemplos claros). En este caso la consonante nasal suele pronunciarse como ligeramente alargada, hecho que interpreto como una transferencia de la mora de la vocal elidida a la consonante; transcribo tales nasales como silábicas (54).

\begin{tabular}{|c|c|c|}
\hline /(h)+̂mo/ & ['(h)im imo] & ‘a ella ( $(+/ \widehat{\jmath})$, a él ( $(+)$ \\
\hline /atáPani// & [a'taąn a'taani] & 'tu cabeza' \\
\hline /íno/ & ['i:no 'i:n] ] & ‘a mí (q)' \\
\hline /manițáana/ & [man'ța:n mani'ța:na] & 'habló' \\
\hline
\end{tabular}

A veces el ensordecimiento afecta las vocales en el interior de la palabra, particularmente si éstas se encuentran cercadas por segmentos sordos (por ejemplo, /6otúxiki/ [6o'tuxikijo] 'mi saliva (q)'). En varios casos, en ese mismo ambiente ninguna vocal está presente a pesar de que la composición morfológica de una palabra (o sus cognados en otras variedades del chiquitano) muestra que diacrónicamente una vocal debió estar presente ahí en algún momento (55). En este análisis, se propone que las vocales están presentes en la representación subyacente (morfofonológica) de los respectivos ítems pero no en la fonológica, ya que su elisión en estos casos parece ser categórica e imprevisible. El origen del fenómeno, sin duda, es relacionado al ensordecimiento vocálico: de lo contrario se esperaría que se atestiguara antecediendo segmentos sonoros, lo que no ocurre.

\begin{tabular}{|c|c|c|c|}
\hline \multirow[t]{2}{*}{ |o-kitapaki-xí| } & /oktapakíci/ & [oktapa'kic] & 'anta. $\hat{O}^{\prime \prime}$ \\
\hline & (cf. /kitapakíci/ & [kitapa'kiø] & 'anta. $\left.\varphi^{\prime}\right)$ \\
\hline \multirow[t]{2}{*}{ |o-sutone-xł| } & /ostonźsł̇/ & [osto'nes] & 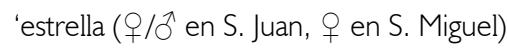 \\
\hline & (cf. /sutonźsł') & [suto'nes] & 'estrella. প’') \\
\hline \multirow[t]{2}{*}{$\mid a-a-r i k i=r \varepsilon\} \varepsilon \mid$} & láski= =rع?દ́/ & ['aşkirg,?? & 'hasta luego (P)' (lit. 'acostate pues') \\
\hline & (cf. /arikíka/ & [ari'kiką] & 'estás acostado') \\
\hline \multirow[t]{2}{*}{$\mid \mathrm{i}_{2}$-ara-ta $=\mathrm{to} \mid$} & /jáşta=f́mo/ & [,jaşta'?̦i:mo] & 'lo botó’ \\
\hline & (cf. /ajára=t́mo/ & [a,jara'?̦i:mo] & 'a botarlo (vos u otro)' \\
\hline
\end{tabular}

Un fenómeno de elisión que sí se atribuye al nivel posléxico afecta las secuencias finales átonas de dos tipos: I-xí, -si, -6i/ (56a) y /-VPV/ (56b). Las secuencias del primer tipo ocurren como alomorfos del sufijo |-xí| × (sustantivo singular, no diminutivo, sin un poseedor referencial) o como partes no segmentables de raíces (como en |-kipoosi| 'esposa'). Las secuencias afectadas del segundo tipo involucran vocales átonas idénticas separadas por una oclusiva glotal; en este caso la vocal final se suele elidir completamente. Si las vocales no son idénticas o si el acento recae en una de ellas, la elisión no ocurre: /f́po?i/ ['ípo?ị] 'adentro', /áuki náPa/ [,aunki'na?å] 'de aquí'. 
a.

b.

\begin{tabular}{|c|c|}
\hline [xiripi'tix] & 'trapo' \\
\hline ['tọ:x] & 'dos' \\
\hline [عtșípơ:s] & 'mi esposa' \\
\hline [si't]عs] & 'su hija' \\
\hline [axtsa'?ৃic] & 'jasayé' \\
\hline [irri'rí & 'su tari' \\
\hline ['taisis] & 'también' \\
\hline [ka'prma?] & 'cafecito' \\
\hline ['ía?] & 'a comer yo' \\
\hline [ta'ßaع̨tso?] & 'es grande' \\
\hline
\end{tabular}

El hecho de que las palabras como aquellas en (56) contienen una vocal final subyacente se hace evidente en el habla fluida (57a), cuando hay sufijación (57b) y en las preguntas de contenido, que presentan un fenómeno de desplazamiento acentual hacia la derecha (57c).

a.

b.

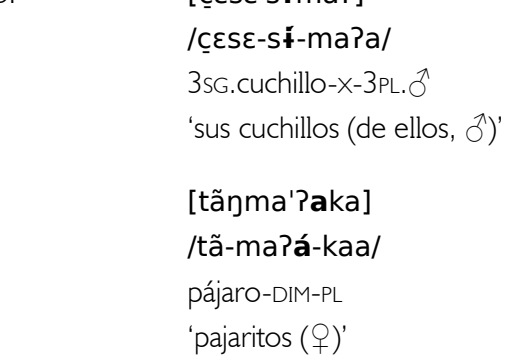

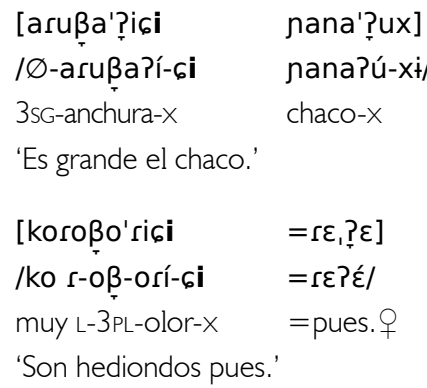

\begin{tabular}{|c|c|c|c|c|c|}
\hline ['irakana,a & & 'ßָatso & tså & tamoko'xi் ] & aislado: [tamo'kơx] \\
\hline /í r-áka & na?á & $\beta$-a-tso & tsa?a & tamokó-xi்' / & \\
\hline qué L-ser.3sG & lo que & 3sG-comer-F.3 & este & perro- $x=?$ & \\
\hline
\end{tabular}

aislado: [arußa'?̦ic]

aislado: [koroßo'ric]

compare: [çع'søs]

'su cuchillo (de ella)'

compare: ['tãnma?]

'pajarito'

Finalmente, menciono algunas aparentes excepciones de las regularidades propuestas arriba.

i. La vocal final del sufijo de plural nominal [-ka] parece ser inmune al ensordecimiento (['tọ:x 'paĩnka] 'dos meses', [tamo'kơka] 'perros'), a diferencia del sufijo de F.SAP /-ka/ [kạ -ka] (['ọ̃nkạ] 'vomitaste', [itjo'kọka] 'estoy bailando'). Se puede explicar este fenómeno representando fonológicamente el sufijo de plural nominal como /-kaa/.

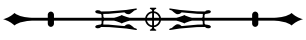


ii. El ítem [ał̣'moxi] 'recién, ahorita' se realiza siempre con una vocal final, sin la esperada elisión (compárese /tamokóxt/ [tamo'kox] 'perro'), lo que se captura representando este ítem como /ałuyóxił/.

iii. Algunas palabras presentan un contorno rítmico inesperado, con una de las sílabas postónicas portando un acento secundario que parece bloquear el ensordecimiento de la vocal final. Como se trata de un número muy reducido de temas, no se propondrá aquí un análisis unificado para estos casos; es posible que el fenómeno tenga alguna relación con la presencia de una vocal alta en la sílaba postónica. Los temas bajo

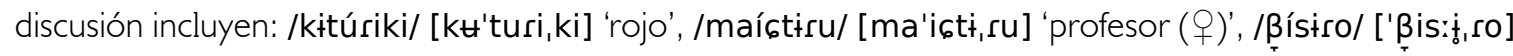
'es recto', /sú́tsaß̧o/ ['sun,tsaß̧o] 'es hediondo' y sus formas flexionadas.

\section{SILABIFICACIÓN ${ }^{21}$}

Las secuencias de dos vocales pueden ser tautosilábicas o heterosilábicas. La silabificación es casi siempre predecible, con poquísimas excepciones (['saį ] 'seis', ['paĩx] 'mes'; cf. [ßָa. 'í] 'curichi', [a. 'ix] 'su hijo') que pueden ser atribuidas a un patrón acentual excepcional. Obsérvese que el acento en chiquitano no es previsible y cada morfema posee su propio patrón acentual subyacente.

Las secuencias de vocales idénticas son tautosilábicas, esto es, se realizan como vocales largas: /toóxi// ['tơ:x] 'dos', /ß̧aáxt/ ['ß̧a:x] 'rocío'. Las analizo como secuencias de segmentos y no como unidades monosegmentales porque no se hallaron evidencias que apuntaran a lo contrario, mientras que el análisis bisegmental permite explicar la realización de formas morfológicamente complejas como |a-a-ka| /aáka/ ['a:.ką] 'comiste' sin recurrir a reglas adicionales.

Las secuencias de vocales distintas en que la segunda vocal es más alta que la primera suelen ser tautosilábicas,

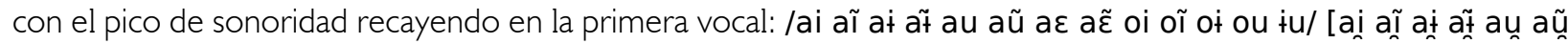

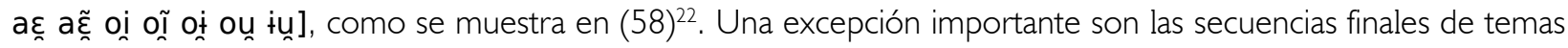
nominales, que se suelen silabificar como heterosilábicas (59).

\begin{tabular}{|c|c|c|}
\hline /apataß̦áiça/ & [a.pa.ta.'ßָai.ccą] & 'ustedes matan' \\
\hline /máĩkỉ̉o/ & ['maĩn.kij.?o] & 'pidió' \\
\hline /mainonițáana/ & [maí.no.ni.'ța:.ṇ] & 'enseñó’ \\
\hline /mapát̃?a/ & [ma.'paĩ.?ą] & 'mintió' \\
\hline /auki/ & [aun.ki] & 'de (ablativo)' \\
\hline /maũxókono/ & [maũny.'xọ.ko.no] & 'está enfermo' \\
\hline /marmatakúrutso/ & [maz..ma.ta.'ku.ru.tso] & 'está esperando' \\
\hline /az̃táma?a/ & [ačn.'ta.ma?] & 'solito (vos u otro)' \\
\hline /oițîtimáxił/ & [oi..tit.'mix] & 'tigre (ぷ')' \\
\hline /țiłmóĩțaPa/ & [țjij.'moĩnni.țjap] & 'en el medio de' \\
\hline /ołmóxit/ & [o+..'mox] & 'urina $($ ぶ')' \\
\hline /taijóuko/ & [tai..joun..ko] & 'está volcando' \\
\hline
\end{tabular}

21 En esta sección indico la silabificación en las transcripciones fonéticas, mediante un punto.

${ }^{22}$ Un(a) evaluador(a) anónimo/a indaga si las secuencias vocálicas tautosilábicas no podrían ser analizadas como diptongos. Aquí se prefirió un análisis que involucra secuencias de fonemas — y no diptongos_ - porque no se han observado restricciones de coocurrencia de segmentos vocálicos en el chiquitano migueleño y porque en algunos casos los elementos que constituyen las secuencias vocálicas pertenecen a morfemas distintos (por ejemplo, /ołuyóxi/ 'urina (đđ)' contiene el prefijo /o-/, que marca el género no humano animado en el habla masculina, y la raíz /łuyo-/ 'urina'). Véase Sánchez Miret (1998) para una discusión de los problemas asociados al uso del término 'diptongo'. 
(59)

\begin{tabular}{|c|c|c|}
\hline /ß̦aí-бi/ & [ßָa.'ic] & 'curichi' \\
\hline /દ́-ts-àt/ & ['z.tsa.ti] & 'mi hijo' \\
\hline /tamaĩ-mara/ & [ta.ma. ̃n.ma?] & 'chichita' \\
\hline /pakaú-kaa/ & [pa.ka.'u.ka] & 'plátanos' \\
\hline /6oú-xí/ & [60.'ux] & 'culebra, víbora (P)' \\
\hline /prí-6i// & [pe.'i६] & 'peji (ج)' \\
\hline
\end{tabular}

Las secuencias de dos vocales distintas en que la segunda es más baja que la primera son tautosilábicas, pero con el pico de sonoridad recayendo en la segunda vocal: /o

$(60)$

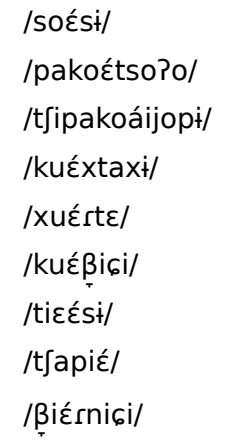

\begin{tabular}{|c|}
\hline \multirow{2}{*}{$\begin{array}{l}\text { ['socs] } \\
\text { [pa. 'ko }\end{array}$} \\
\hline \\
\hline [t]i.pa.'konai.jo.pịt] \\
\hline ['kucx.tax] \\
\hline ['xungr.teg] \\
\hline ['kunع.ßָi६ ] \\
\hline ['tik:s:s] \\
\hline [t]a.'pir $\varepsilon]$ \\
\hline ['ßiંદr.ni६] \\
\hline
\end{tabular}

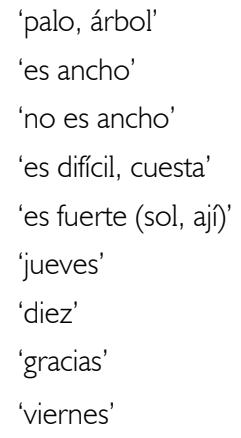

La silabificación de la secuencia /łu/, la única que involucra vocales de la misma altura, parece depender de la posición del acento subyacente. Si se acentúa la primera mora, la realización parece ser [iự] ([tuu] en San Juan), como en

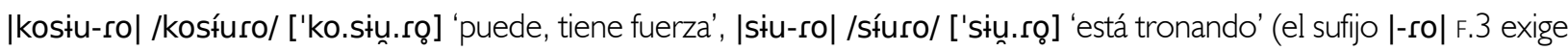
que el acento recaiga en la antepenúltima mora). En cambio, si es la segunda mora que se acentúa, la secuencia /łu/ parece

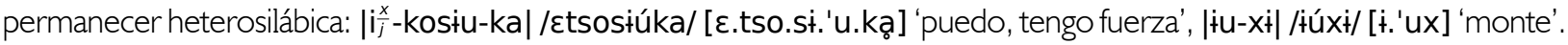

\section{EPÉNTESIS DE [jo]}

El último proceso, posléxico y opcional, afecta las palabras que terminan en /i/, sin importar si ésta es tónica o átona, y consiste en una inserción de la secuencia [jo jo ço] en el margen derecho de la palabra fonológica. Algunos ejemplos (tanto extraídos de diálogos espontáneos como elicitados) se dan en (61).

\begin{tabular}{|c|c|c|c|}
\hline [icî̃̃jaka & naĩn'kijo] & \multicolumn{2}{|c|}{ [,kãatatu'ruß̧įço] ] } \\
\hline /i-ciná-ka & n-a-ĩkí/ & /...kápa & a-taturúßii/ \\
\hline 1sG.ðे-querer-F.SAP & 1sG. $\hat{0}$-ACT-preguntar & ...para_que & 2/3sG.NF.ACT-enjuagar \\
\hline 'Quiero preguntar $\left(\jmath^{\Uparrow}\right) . '$ & & \multicolumn{2}{|c|}{ ‘[El cepillo] para enjuagarse.’ } \\
\hline 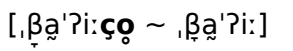 & [6o'tuxijkijo] & [ko'rii $r \varepsilon, \varepsilon$ & ß̧atu'ßָaxiço] \\
\hline /ßָápa $\varnothing$-íi/ & /6-otúxiki/ & /korípi= rع? & $\beta$-a-tußáxi/ \\
\hline estos. +1 ss.. -canilla & 1sG. O-saliva & HORT $=$ pues. $q$ & 1INCL-ACT-moler \\
\hline 'Mis canillas (q).' & 'Mi saliva.' & 'Vamos a mole & \\
\hline
\end{tabular}




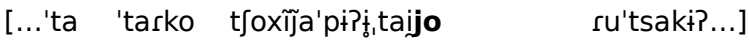 \\ /...tá táruko t -oxina-pípi=tái r-u-ts-á-k-ipł.../ \\ FOC muy NEG-bueno-NEG=nomás L-1INCL-TH-estar-F.SAP-PSTV}

'[Cuando estamos solas,] es muy mal nomás que estemos [así, cuando nos enfermamos].'

Ningún fenómeno semejante se ha atestiguado en las demás variedades del chiquitano y tampoco se sabe cuál motivación podría subyacerlo.

\section{CONCLUSIONES}

En este artículo se presentó una primera descripción de la fonología segmental del chiquitano migueleño. Con ello, se pretende contribuir a la documentación de esta variedad lingüística severamente amenazada. Los fenómenos que podrían presentar interés tipológico incluyen la palatalización progresiva, la realización variable de las vocales nasales, el ensordecimiento y la elisión de vocales en determinados ambientes.

La palatalización total de las consonantes labiales $(\mid \mathrm{p} \beta \mathrm{m} \mathrm{m} \rightarrow / \underline{\mathrm{c}} \mathrm{n} /)$, en particular, no ha sido previamente atestiguada en las lenguas de la región chaqueña y en zonas adyacentes ( $c f$. González, 2014) ni en los demás dialectos del chiquitano, en los que las consonantes labiales pueden sufrir palatalización secundaria —por ejemplo, /p m/ $\rightarrow\left[p^{j} \mathrm{~m}^{\mathrm{j}}\right]$ en el bésiro, ver Sans (2010, p. 103)—, pero no total. Efectivamente, se trata de un patrón muy raro translingüísticamente: en una muestra de 117 lenguas analizada por Bateman (2007), apenas dos presentan el patrón de la palatalización total —en oposición a la secundaria — de los segmentos labiales: el dialecto del rumano hablado en la Moldavia rumana (romance, familia indoeuropea) y el setsuana (bantú, familia Atlántico-Congo). En ambos casos, las evidencias comparativas, morfofonológicas y filológicas convergen en señalar que la palatalización total de los segmentos labiales en esas variedades es esencialmente epifenomenal, ya que los respectivos cambios fonéticos involucraron etapas adicionales, tales como la fortición de una semivocal palatal y la elisión o la absorción del segmento labial original (por ejemplo, $b>b j>b j>b g^{j}>g^{j}$; ver Bateman, 2007, p. 89). La autora hipotetiza que las secuencias de cambios fonéticos de este tipo (es decir, las que involucran secuencias bisegmentales) son el único mecanismo diacrónico capaz de engatillar la palatalización total de una consonante labial. En este sentido, el chiquitano migueleño podría resultar problemático para la hipótesis de Bateman (2007), ya que esta variedad no presenta ninguna evidencia

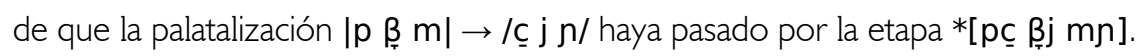

Por razones de espacio, no se han podido abordar temas como la estructura silábica, la fonética y la fonología del acento y los procesos morfofonológicos activos en el chiquitano migueleño (con la excepción de la palatalización). Estos temas se discuten parcialmente en un otro trabajo (Nikulin, 2020b), basado en el mismo corpus.

Se espera que las generalizaciones expuestas aquí permitan mejorar la propuesta ortográfica de Nikulin (2019a) para el chiquitano migueleño, que sufre de ciertas inconsistencias, y que contribuyan para la comprensión de la diversidad lingüística de la Chiquitanía.

\section{GLOSARIO}

En este glosario se reproducen los términos regionales (del castellano camba) que ocurren en las glosas de este artículo y se proporcionan definiciones. Para los zoónimos y fitónimos, se dan sus equivalentes en portugués y en el castellano venezolano (dos otras variedades ampliamente conocidas por los estudiosos de la Amazonía). 


\begin{tabular}{|c|c|c|c|c|c|}
\hline Camba & \multicolumn{2}{|c|}{ Definición } & Camba & \multicolumn{2}{|c|}{ Definición } \\
\hline Apero & \multicolumn{2}{|c|}{$\begin{array}{l}\text { Parte de la silla de montar que tiene los } \\
\text { dos arzones }\end{array}$} & Jone & \multicolumn{2}{|c|}{ Pedazo de ladrillo, adobe } \\
\hline Chaco & \multicolumn{2}{|c|}{ Conuco } & Paúro & \multicolumn{2}{|c|}{ Pozo de agua } \\
\hline Chompa & \multicolumn{2}{|c|}{ Suéter } & Puchi & \multicolumn{2}{|c|}{ Heces } \\
\hline Curichi & \multicolumn{2}{|c|}{ Barrizal, lugar anegado } & Tacú & \multicolumn{2}{|c|}{ Mortero } \\
\hline Jachi & \multicolumn{2}{|c|}{ Residuo de chicha } & Tapeque & \multicolumn{2}{|c|}{ Provisión de víveres para un viaje } \\
\hline Jasayé & \multicolumn{2}{|c|}{ Tipo de cesto tradicional } & Tari & \multicolumn{2}{|c|}{ Tipo de recipiente tradicional } \\
\hline \multicolumn{6}{|c|}{ Animales } \\
\hline Camba & Venezolano & Portugués & Camba & Venezolano & Portugués \\
\hline Capiguara & Chigüire & Capivara & Peto & Abeja silvestre & Caba \\
\hline Ca(s)caré & Especie de piarro & $\begin{array}{l}\text { Gralha-do- } \\
\text { pantanal }\end{array}$ & Seboí & Tordo & Chupim \\
\hline Chuubi & Gavilán & Gavião & Sepe & Comején & Cupim \\
\hline Cujuchi & Especie de roedor & Tuco-tuco & Sucha & Zamuro & Urubu \\
\hline Corechi & Especie de cachicamo & Tatu-bola & Tapití & Conejo de monte & Tapiti \\
\hline Macono & Halcón macagua & Acauã & Tarechi & Loro hablador & Maitaca-verde \\
\hline Masi & Ardilla & Serelepe & Tatú & Cachicamo & Tatu \\
\hline Peji & Especie de cachicamo & Tatu-peba & Tibibi & Tigüi-tigüe & Perna-amarela \\
\hline Peni/jaúsi & Mato & Calango/teiú & Turu & Caracol & Caracol \\
\hline Peta & Morrocoy & Jabuti & Urina & Venado & Veado \\
\hline \multicolumn{6}{|c|}{ Plantas } \\
\hline Almendra & $\begin{array}{l}\text { Nuez parecida a la } \\
\text { sarrapia }\end{array}$ & Cumaru & Isotoúbo & Paraparo & Saboeiro \\
\hline Azucaró & Jobo & Taperebá & Motoyoé & Mamoncillo & Especie de fruta \\
\hline Bibosi & Matapalo & Gameleira & Paja sujo & Esp. de hierba & Capim-sapé \\
\hline Caracoré & Cardón, cirio & Mandacaru & Paquió & Algarrobo & Jatobá \\
\hline Curupaú & Yopo & Angico & Soriocó & Bucare & Mulungu \\
\hline Garabatá & Maya & Caraguatá & Sucupiro & Alcornoque & Sucupira-preto \\
\hline Gualele & Cambur & $\begin{array}{l}\text { Espécie de } \\
\text { banana }\end{array}$ & Urucú & Onoto & Urucum \\
\hline Güembé & Especie de bejuco & Cipó-imbé & & & \\
\hline
\end{tabular}

\section{AGRADECIMIENTOS}

Agradezco a Doña Ignacia (Násiya) Yopié Tomichá, a Doña Antonia (Ato'ĩxh) Socoré Masaí, a Don Victoriano Julián Laverán Ramos (in memoriam), a Doña Micaela Ribera Montero y a Don Miguel Putaré Tapanaché (in memoriam) por haberme enseñado su idioma, así como al cacique Elmar Socoré Casupá y a Rubén Pitigá Socoré por el respaldo logístico en San Juan de Lomerío. Expreso mi gratitud a dos evaluadores/as anónimos/as, a Andrés Pablo Salanova y al Editor Asociado de este Boletim, Hein van der Voort, por sus comentarios y sugerencias. Mi conocimiento del 
chiquitano se benefició enormemente de las conversaciones con Brittany Hause y Luca Ciucci, a quienes les doy mis gracias. Además, agradezco la colaboración y compañía de Rafael Andrade, Bruno L’Astorina y Mauricio Meza durante el trabajo de campo en 2017-2018. Finalmente, expreso mi gratitud a Severino Parzinger por haberme proporcionado datos preliminares sobre los hablantes del chiquitano migueleño fuera de San Juan de Lomerío, que posteriormente he podido constatar, aunque parcialmente, in loco. La responsabilidad por cualquier error recae únicamente en mí.

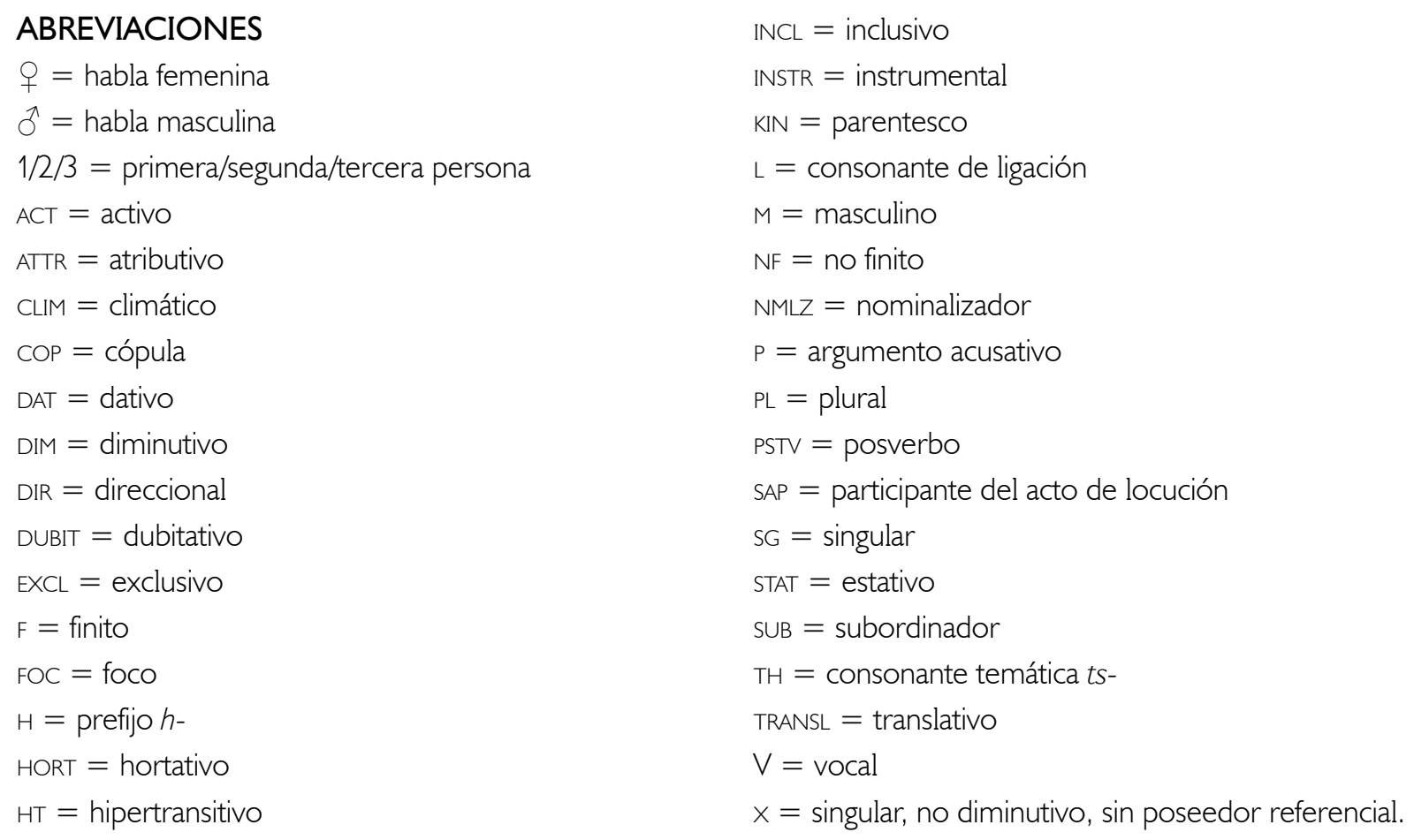

\section{REFERENCIAS}

Adelaar, W. F. H. (2008). Relações externas do Macro-Jê: o caso do Chiquitano. In S. V. T. A. P. L. Telles \& A. S. Paula (Orgs.), Topicalizando Macro-Jê (pp. 9-28). Recife: Nectar.

Álvarez, J. (2016). Esbozo de una gramática de la lengua kari'ña. Recuperado de https://www.academia.edu/28516773/Esbozo_de_una_ gram\%C3\%A1tica_de_la_lengua_kari\%C3\%B1a

Bas Aguilera, B. (sin fecha). Vocabulario de Benjamín Bas. San Miguel de Velasco. Manuscrito.

Bateman, N. (2007). A crosslinguistic investigation of palatalization (Tesis de doctorado). University of California San Diego, San Diego.

Brandão, A. P. B. (2014). A reference grammar of Paresi-Haliti (Arawak) (Tesis de doctorado). University of Texas at Austin, Austin.

Cáceres, N. (2011). Grammaire fonctionnelle-typologique du ye'kwana, langue caribe du Venezuela (Tesis de doctorado). Université Lumière Lyon 2, Lyon.

Ciucci, L., \& Macoñó Tomichá, J. (2018). Diccionario básico del chiquitano del Municipio de San Ignacio de Velasco. Santa Cruz de la Sierra: Industria Maderera San Luis S. R. L.

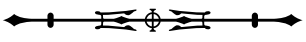


Gafos, A. I. (2002). A Grammar of Gestural Coordination. Natural Language \& Linguistic Theory, 20, 269-337. doi: https://doi. org/10.1023/A:1014942312445

Girard, R. (2012). Documentación de la cantidad vocálica en chiquitano: algunas observaciones preliminares. In H. A. González \& B. Gualdieri (Eds.), Lenguas indígenas de América del Sur I. Fonología y léxico (pp. 25-38). Mendoza: Editorial FFyL-UNCuyo y SAL.

Girard, R. (2014, mayo). Agreement without similarity: the case of Chiquitano nasal (consonant) harmony. University of California-Berkeley, Berkeley. Recuperado de http://inguistics.berkeley.edu/ inkelas/PosterAbstracts/Girard.pdf

González, H. A. (2014). Procesos fonológicos como rasgos areales: el caso de la palatalización en las lenguas chaqueñas. LIAMES, 14(1), 11-39. doi: https://doi.org/10.20396/liames.v0i14.1519

Krüsi, M., \& Krüsi, D. (1978). Phonology of Chiquitano. In Work papers of the Summer Institute of Linguistics Riberalta, Bolivia $1972-1976$ (pp. 53-93). Riberalta: Summer Institute of Linguistics.

Ladefoged, P., \& Maddieson, I. (1996). The sounds of the world's languages. Oxford: Blackwell.

Nikulin, A. (2019a). iManityaka au r-ózura! Diccionario básico del chiquitano migueleño: el habla de San Miguel de Velasco y de San Juan de Lomerío. Recuperado de https://www.academia.edu/38284134/Manityaka_au_r_\%C3\%B3zura_Diccionario_b\%C3\%A1sico_del chiquitano_miguele\%C3\%B1o_El_habla_de_San_Miguel_de_Velasco_y_de_S̄an Juan_de_Lomer\%C3\%AD̄o

Nikulin, A. (2019b). First person singular markers in Migueleño Chiquitano. In D. K. E. Reisinger \& R. Yu-Hsiang Lo (Eds.), Proceedings of the Workshop on the Structure and Constituency of Languages of the Americas 23 (pp. 62-76). Vancouver: UBCWPL.

Nikulin, A. (2019c). Indexical gender and grammatical gender in Chiquitano. Typology of Morphosyntactic Parameters, 2(1), 86-99.

Nikulin, A. (2020a). Proto-Macro-Jê: um estudo reconstrutivo (Tesis de doctorado). Universidade de Brasília, Brasilia, DF.

Nikulin, A. (2020b). Elementos de la morfofonología del chiquitano migueleño. LIAMES, 20, 1-21. doi: https://doi.org/10.20396/liames. v20i0.8660822

Parapaino Castro, P. (2008). Isiukiché nikorokó Bésiro: guía de escritura del idioma Bésiro. Santa Cruz de la Sierra: Unión de Artesanos de la Tierra-UNIARTE.

Parzinger, S. (2017). Los sermones chiquitanos: catequesis ancestral en una sociedad globalizada. Verbum SVD, 58(1), 62-77.

Parzinger, S., \& Cabildo Indígena de San Miguel de Velasco (Eds.). (2016). Osuputakai rurasti Tupáj: Manual de Sermones Chiquitanos de San Miguel de Velasco y sus comunidades (Misión y Diálogo, No. 15). Cochabamba: Editorial Verbo Divino.

Sánchez Miret, F. (1998). Some reflections on the notion of diphthong. In Papers and Studies in Contrastive Linguistics (No. 34, pp. 27-51). Poznan, Poland: School of English, Adam Mickiewicz University.

Sans, P. (2010). Éléments de sociolinguistique et de phonologie du béstro (chiquitano): langue en danger des basses terres de Bolivie (Tesis de maestría). Université Lumière Lyon 2, Lyon.

Sans, P. (2011). Is nasality an autosegmental feature in Béstro (a.k.a. Chiquitano)? In A. S. A. C. Cabral, A. D. Rodrigues, J. D. Lopes \& M. R. S. Julião (Orgs.), Línguas e culturas Tupí (Vol. 2, pp. 237-248). Campinas: Curt Nimuendajú.

Sans, P. (2013). Elementos de la gramática del Béstro: sociolingüística-fonología-morfología-Textos. Recuperado de https://www.academia. edu/4534429/Elementos de la gram\%C3\%A1tica_del_B\%C3\%A9s\%C9\%A8ro. Socioling\%C3\%BC\%C3\%ADstica_Fonolog\%C3\%ADa_-_Mörfolog\%C3\%ADa_-_Textos

Santana, Á. C. (2005). Transnacionalidade lingüística: a língua Chiquitano no Brasil (Tesis de maestría). Universidade Federal de Goiás, Goiânia, GO.

Santana, Á. C. (2012). Línguas cruzadas, histórias que se mesclam: ações de documentação, valorização e fortalecimento da língua Chiquitano no Brasil (Tesis de doctorado). Universidade Federal de Goiás, Goiânia, GO.

Zonneveld, W. (1978). A formal theory of exceptions in phonology. Lisse: Peter de Ridder Press. doi: https://doi.org/10.1515/9783110859911

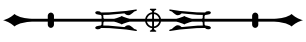

